\title{
1+1 Gaudin Model
}

Andrei V. ZOTOV

Institute of Theoretical and Experimental Physics, Moscow, Russia

E-mail: zotov@itep.ru

Received January 29, 2011, in final form July 03, 2011; Published online July 13, 2011

doi:10.3842/SIGMA.2011.067

\begin{abstract}
We study 1+1 field-generalizations of the rational and elliptic Gaudin models. For $\operatorname{sl}(N)$ case we introduce equations of motion and L-A pair with spectral parameter on the Riemann sphere and elliptic curve. In sl(2) case we study the equations in detail and find the corresponding Hamiltonian densities. The $n$-site model describes $n$ interacting LandauLifshitz models of magnets. The interaction depends on position of the sites (marked points on the curve). We also analyze the 2 -site case in its own right and describe its relation to the principal chiral model. We emphasize that $1+1$ version impose a restriction on a choice of flows on the level of the corresponding $0+1$ classical mechanics.
\end{abstract}

Key words: integrable systems; field theory; Gaudin models

2010 Mathematics Subject Classification: 14H70; 33E05; 37K20; 37K10

\section{Introduction}

Gaudin model (or Gaudin magnet) was introduced by M. Gaudin [1] as a quasiclassical limit of spin-1/2 chain and was studied via the Bethe ansatz [2,3]. Let us start with a general rational model underlying Gaudin magnets. The classical rational Gaudin model is defined by the following quadratic Hamiltonians:

$$
H_{a}=-\frac{1}{2} \sum_{c \neq a} \frac{\left\langle S^{a} S^{c}\right\rangle}{z_{a}-z_{c}}, \quad a=1, \ldots, n,
$$

where $S^{a} \in \operatorname{sl}(2, \mathbb{C}),\left\{z_{1}, \ldots, z_{n}\right\} \in \mathbb{C P}^{1}$ are marked points and \langle\rangle denotes the trace.

From the point of view of the Lax pair the model is described by a general Lax matrix which is a $\operatorname{sl}(N, \mathbb{C})$-valued function $L(z)$ on $\mathbb{C P}^{1} \backslash\left\{z_{1}, \ldots, z_{n}\right\}$ with simple poles at $\left\{z_{1}, \ldots, z_{n}\right\}$ and some given residues $\operatorname{Res}_{z_{a}} L(z)=S^{a} \in \operatorname{sl}(N, \mathbb{C})$ :

$$
L(z)=\sum_{a=1}^{n} \frac{S^{a}}{z-z_{a}} .
$$

The generating function of the Hamiltonians is

$$
\frac{1}{2}\left\langle L^{2}(z)\right\rangle=\frac{1}{2} \sum_{a=1}^{n} \frac{\left\langle\left(S^{a}\right)^{2}\right\rangle}{\left(z-z_{a}\right)^{2}}+\sum_{a \neq b} \frac{1}{z-z_{a}} \frac{\left\langle S^{a} S^{b}\right\rangle}{z_{a}-z_{b}} .
$$

The first sum in (1.3) shows that the eigenvalues $\lambda_{a}$ of $S^{a}$ are the constant $\mathbb{C}$-numbers. Thus, the phase space is a direct product ${ }^{1}$ of the coadjoint orbits by $\operatorname{SL}(N, \mathbb{C})$ action: $M=\mathcal{O}^{1} \times$

\footnotetext{
${ }^{1}$ In fact, there is coadjoint action of $\operatorname{SL}(N, \mathbb{C})$ on $\mathrm{M}$ which provides the constraint $\sum_{a} S^{a}=0$ with some fixation of $\operatorname{SL}(N, \mathbb{C})$ action. Then one can make a reduction $M \rightarrow M / / \operatorname{SL}(N, \mathbb{C})$. But we do not go into details of this reduction here. In $[4,5]$ the examples of the reduction for the Painlevé VI equation are discussed. The $r$-matrix of the reduced models satisfies the reflection equations. Thus, the models live on the boundaries of the finite lattices.
} 
$\cdots \times \mathcal{O}^{n}$. This phase space is naturally equipped with a linear Poisson-Lie structure:

$$
\left\{S_{\alpha}^{a}, S_{\beta}^{b}\right\}=\delta^{a b} \sum_{\gamma} C_{\alpha \beta}^{\gamma} S_{\gamma}^{a},
$$

where $S_{\alpha}^{a}$ are coefficients in some basis $\left\{T_{\alpha}\right\}: S^{a}=\sum_{\alpha} S_{\alpha}^{a} T_{\alpha}$ and $C_{\alpha \beta}^{\gamma}$ are the structure constants of $\operatorname{sl}(N, \mathbb{C})$ in this basis. The natural basis is described in the appendix. The Hamiltonians (1.1) in $\operatorname{sl}(N, \mathbb{C})$ case are replaced by

$$
H_{a}=-\frac{1}{N} \sum_{c \neq a} \frac{\left\langle S^{a} S^{c}\right\rangle}{z_{a}-z_{c}}, \quad a=1, \ldots, n
$$

The dynamics with respect to the Hamiltonians (1.5) is given by the following equations ${ }^{2}$ :

$$
\begin{aligned}
& \partial_{t_{a}} S^{a}=\left\{H_{a}, S^{a}\right\}=-\sum_{c \neq a} \frac{\left[S^{a}, S^{c}\right]}{z_{a}-z_{c}}, \\
& \partial_{t_{a}} S^{b}=\left\{H_{a}, S^{b}\right\}=\frac{\left[S^{a}, S^{b}\right]}{z_{a}-z_{b}} \quad \text { for } \quad a \neq b .
\end{aligned}
$$

These equations of motion can be represented in the Lax form

$$
\partial_{t_{a}} L=\left[L, M_{a}\right]
$$

with the Lax pair

$$
L(z)=\sum_{c=1}^{n} \frac{S^{c}}{z-z_{c}}, \quad M_{a}(z)=\frac{S^{a}}{z-z_{a}} .
$$

In such a generality the model was studied many times. For example, the non-autonomous version corresponds to the Schlesinger system of the isomonodromic deformations on a sphere. It was studied a hundred years ago [6].

In the elliptic case [7] the Lax matrix (1.2) is replaced by

$$
L(z)=\sum_{a=1}^{n} \sum_{\alpha} S_{\alpha}^{a} \varphi_{\alpha}\left(z-z_{a}\right) T_{\alpha},
$$

where $z \in \Sigma_{\tau}$ is a coordinate on an elliptic curve $\Sigma_{\tau}$ with moduli $\tau$. Basis $\left\{T_{\alpha}\right\}$ and the corresponding Poisson structure is defined in (B.4). Functions $\varphi_{\alpha}\left(z-z_{a}\right)$ (B.10) form a basis in $\Gamma$ (End $V, \Sigma_{\tau}$ ) with a simple pole at $z_{a}$ for some fixed holomorphic vector bundle $V$ of degree one. The Poisson structure (1.4) for the structure constants (B.7) is related to the existence of the $r$-matrix of the Belavin-Drinfel'd type [8]. The quadratic Poisson structure can be defined by the same $r$-matrix [9].

Most of problems natural for integrable systems have been studied for the Gaudin model as well. Among them the separation of variables [10], relations to monodromy preserving and Knizhnik-Zamolodchikov equations [11], quantum quadratic algebras and bihamiltonian structures [12], time-discrete versions [13], quantization [14] and Langlands duality [15]. It should be mentioned that the elliptic Gaudin model was originally defined by B. Enriquez and V. Rubtsov [16] as an example of the Hitchin-type system [17]. "Dynamical" case was considered first by A. Gorsky and N. Nekrasov [18]. That case corresponded to degree zero vector bundle $V$ (that is to nontrivial moduli space of bundles) or to the "spin" extensions of the Calogero model.

\footnotetext{
${ }^{2}$ Here we imply some choice of the normalization by the Killing form \langle\rangle , see also (B.6).
} 
In [19] it was shown that the top-like models and Calogero-type models are related by means of the modification procedure (the later changes the degree of $V$ ). In this respect, the models are equivalent.

Consideration of particular cases and different types of reductions leads to relations between Gaudin model and a number of known integrable systems such as interacting tops [20], Painlevé VI equation and Zhukovsky-Volterra gyrostat [4], Neumann system [21].

With the advent of the inverse scattering method the Lax equations or the zero-curvature equations [22] (with spectral parameter) became a main tool for investigation of nonlinear equations [23, 24]. Different applications and classifications can be found in [25]. In this paper we are predominantly interested in the Landau-Lifshitz equation [26] (which describes the continuous limit of the XYZ model $[27,28])$ and the principal chiral model $[29,30]$.

In [19] a general scheme was suggested for constructing $1+1$ (or field) generalizations of the Gaudin-type models as typical examples of the Hitchin systems. As a by-product of this work the field generalization of the elliptic Calogero model was obtained ${ }^{3}$ and its equivalence to the Landau-Lifshitz equation was shown in terms of the special singular gauge transformations.

The purpose of the paper is to present explicit L-A pairs for $1+1$ Gaudin model, to propose corresponding Hamiltonian description and to find out relationships between the obtained equations and some known models such as the Heisenberg Model, the Landau-Lifshitz equation and the principal chiral model.

In 1+1 models the Lax equations (1.6) are replaced by the zero-curvature (or ZakharovShabat) equations:

$$
\partial_{t_{a}} L-\partial_{x} M_{a}=\left[L, M_{a}\right],
$$

where $L$ and $M_{a}$ do not coincide (in general) with those from (1.6). It was shown in [19] how to construct $1+1$ version of $L$-operator. In particular, $L$ keeps the same form as in $(0+1)$ version of the Hitchin systems corresponding to holomorphic vector bundles of degree 1 . This class of systems is under our consideration in this paper. A general scheme [32] allows to obtain densities of the conserved quantities (Hamiltonians). However, there is a technical problem of finding corresponding $M$-operators. Unfortunately, there is no practical way to get them explicitly. For example, in [19] the nontrivial $M$-operator for the field version of Calogero model was obtained by some ansatz. In the same manner $M$-operators were obtained in $[27,30]$ for the Landau-Lifshitz and the Principal Chiral Models correspondingly. The inverse problem (to find mechanical $L$ and $M$ from known field versions) is an easy task - one should put to zero all derivatives with respect to the loop variable $x$. In this respect, there is a correspondence between field flows and some choice of flows ( $M$-operators) on the level of classical mechanics. It will be shown that the first flows of $1+1$ Gaudin hierarchy correspond to "conventional" description of flows in the Gaudin mechanics while the second flows arise naturally from some "reformulated" version. The later appears as some linear combination of the "conventional" Gaudin flows.

The paper is organized as follows: in Section 2 we give a standard description of the Gaudin model and its flows $M_{a}$ (Proposition 2.1). Then the "reformulated" version is suggested in the form of linear combinations of $\left\{M_{a}\right\}$ (Proposition 2.2). In Section 3 we discuss the field generalization and find the first (Proposition 3.1) and the second (Proposition 3.2) flows of the 1+1 Gaudin hierarchy. Among other things, we consider a special case of the first flows corresponding to the principal chiral model in detail. In Section $4 \operatorname{sl}(2, \mathbb{C})$ case is considered (rational - Subsection 4.1 and elliptic - Subsection 4.2) and the Hamiltonian description is obtained. First, we get general formulae for the densities of Hamiltonians via local decomposition for the first (Lemma 4.1) and the second (Lemma 4.2) flows. Secondly, we evaluate these densities for $1+1$ rational and elliptic $\operatorname{sl}(2, \mathbb{C})$ Gaudin model and reproduce previously obtained equations of motion (Theorem 4.1).

\footnotetext{
${ }^{3}$ This result was first obtained by I. Krichever in [31].
} 
The results of the paper can be briefly summarized as follows:

$(0+1)$ mechanics:

Gaudin flows $\left\{H_{a}\right\}$

Gaudin flows $\left\{\tilde{H}_{a}\right\}$

("reformulated version")

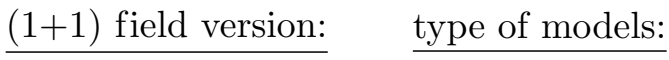

$1^{\text {st }}$ flows $\left\{\mathcal{H}_{a, 1}\right\}$

$2^{\text {nd }}$ flows $\left\{\mathcal{H}_{a, 2}\right\}$ $n$-site generalization of

principal chiral model

interacting models of

Landau-Lifshitz type

The first flows are described by the following equations:

$$
\begin{aligned}
& \partial_{t_{a}} S^{a}-k \partial_{x} S^{a}=-\sum_{c \neq a}\left[S^{a}, \hat{\varphi}_{a c}\left(S^{c}\right)\right], \\
& \partial_{t_{a}} S^{b}=\left[S^{b}, \hat{\varphi}_{b a}\left(S^{a}\right)\right] .
\end{aligned}
$$

In "2-site" case and rational limit these are the equations of the principal chiral model:

$$
\begin{aligned}
& \partial_{t} l_{1}-k \partial_{x} l_{0}+\frac{2}{z_{1}-z_{2}}\left[l_{1}, l_{0}\right]=0, \\
& \partial_{t} l_{0}-k \partial_{x} l_{1}=0
\end{aligned}
$$

with $l_{0}=S^{1}+S^{2}$ and $l_{1}=S^{1}-S^{2}$.

The equations for the second flows are of the form (here we put $\operatorname{sl}(2, \mathbb{C})$ case and $\operatorname{sl}(N, \mathbb{C})$ is considered below):

$$
\begin{aligned}
& \partial_{\tilde{t}_{a}} S^{a}-k \partial_{x} \eta^{a}=\left[S^{a}, \hat{\wp}\left(S^{a}\right)\right]+\sum_{c \neq a}\left[\eta^{a}, \hat{\varphi}_{c a}\left(S^{c}\right)\right]-\left[\hat{F}_{c a}\left(S^{c}\right), S^{a}\right], \\
& \partial_{\tilde{t}_{a}} S^{b}=\left[\hat{\varphi}_{a b}\left(\eta^{a}\right), S^{b}\right]+\left[S^{b}, \hat{F}_{b a}\left(S^{a}\right)\right],
\end{aligned}
$$

where $\eta^{a}=-\frac{k}{4 \lambda_{a}^{2}}\left[S^{a}, S_{x}^{a}\right]+\sum_{c \neq a} \hat{\varphi}_{a c}\left(S^{c}\right)$. Note that in "1-site" case $n=1$ the first one equation in (1.8) is the Landau-Lifshitz equation (for $t_{1}=t$ ):

$$
\partial_{t} S+\frac{k^{2}}{4 \lambda^{2}}\left[S, S_{x x}\right]=[S, \hat{\wp}(S)] .
$$

\section{$2 \operatorname{sl}(N, \mathbb{C})$ elliptic Gaudin model}

\subsection{Standard description}

The phase space of the Gaudin model is a direct product of orbits $\mathcal{O}_{1} \times \cdots \times \mathcal{O}_{n}$ by the coadjoint action of $\operatorname{SL}(N, \mathbb{C}))$. The coordinates $\left\{S_{\alpha}^{c}\right\}$ on each orbit $S^{c} \in \mathcal{O}_{c}$ are chosen to be dual to the basis $\left\{T_{\alpha}\right\}$ of the Lie algebra $\operatorname{sl}(N, \mathbb{C})$. The later basis $\left\{T_{\alpha}\right\}$ is built as the projective representation of $(\mathbb{Z} / N \mathbb{Z} \oplus \mathbb{Z} / N \mathbb{Z})$ in $\mathrm{GL}(N, \mathbb{C})$ (see (B.4)). The corresponding structure constants (B.7) provides the Poisson-Lie brackets:

$$
\left\{S_{\alpha}^{a}, S_{\beta}^{b}\right\}=\delta^{a b} c_{\alpha, \beta} S_{\alpha+\beta} .
$$

Let us introduce now the Lax matrix defined on the elliptic curve $\Sigma_{\tau}=\mathbb{C} /(\mathbb{Z}+\tau \mathbb{Z})$ with modular parameter $\tau(\operatorname{Im}(\tau)>0)$ :

$$
L(z)=\sum_{c=1}^{n} \sum_{\alpha \in \Gamma^{\prime}} S_{\alpha}^{c} T_{\alpha} \varphi_{\alpha}\left(z-z_{c}\right),
$$


where $\Gamma_{N}^{\prime}=\tilde{\mathbb{Z}}_{N}^{(2)}$ (see (B.3)) and functions $\left\{\varphi_{\alpha}\left(z-z_{c}\right)\right\}$ form the basis in the space of sections $\Gamma$ (End $\left.V, \Sigma_{\tau}\right)$ with simple poles at $\left\{z_{c}\right\}, c=1, \ldots, n$ for the holomorphic vector bundle $V$ of degree one associated with the principle $\operatorname{GL}(N, \mathbb{C})$-bundle over $\Sigma_{\tau}$. In fact the Lax matrix is fixed by the quasiperiodic properties with (B.1), (B.2):

$$
L(z+1)=Q L(z) Q^{-1}, \quad L(z+\tau)=\Lambda L(z) \Lambda^{-1}
$$

and residues $\operatorname{Res}_{z_{a}} L(z)=S^{a}$.

The invariants of the Lax matrix generate commuting Hamiltonians ${ }^{4}$

$$
\frac{1}{2 N}\left\langle L^{2}(z)\right\rangle=\sum_{c=1}^{n}\left(H_{2, c} \wp\left(z-z_{c}\right)-H_{1, c} E_{1}\left(z-z_{c}\right)\right)-H_{0},
$$

where $H_{2, c}=\frac{1}{2 N}\left\langle\left(S^{c}\right)^{2}\right\rangle=\frac{1}{2} \sum_{\alpha \in \Gamma_{N}^{\prime}} S_{\alpha}^{c} S_{-\alpha}^{c}$ are the Casimir functions corresponding to the orbits $\mathcal{O}_{c}$ and the Hamiltonians are:

$$
\begin{aligned}
H_{1, a} & =-\frac{1}{N} \sum_{c \neq a}\left\langle S^{a} \hat{\varphi}_{a c}\left(S^{c}\right)\right\rangle=-\sum_{c \neq a} \sum_{\alpha \in \Gamma_{N}^{\prime}} S_{-\alpha}^{a} S_{\alpha}^{c} \varphi_{\alpha}\left(z_{a}-z_{c}\right), \\
H_{0} & =\frac{1}{2 N} \sum_{c}\left\langle S^{c} \hat{\wp}\left(S^{c}\right)\right\rangle-\frac{1}{2 N} \sum_{b \neq c}\left\langle S^{b} \hat{f}_{b c}\left(S^{c}\right)\right\rangle \\
& =\frac{1}{2} \sum_{c} \sum_{\alpha \in \Gamma_{N}^{\prime}} S_{-\alpha}^{c} S_{\alpha}^{c} \wp\left(\omega_{\alpha}\right)-\frac{1}{2} \sum_{b \neq c} \sum_{\alpha \in \Gamma_{N}^{\prime}} S_{-\alpha}^{b} S_{\alpha}^{c} f_{\alpha}\left(z_{b}-z_{c}\right),
\end{aligned}
$$

where we use the following notations: $\wp\left(\omega_{\gamma}\right)$ is defined in (B.8), functions $\varphi_{\gamma}(z)$ and $f_{\gamma}(z)$ in (B.10), (B.11). We also define the linear operators:

$$
\hat{\wp}: S_{\alpha} \rightarrow S_{\alpha} \wp\left(\omega_{\alpha}\right), \quad \hat{\varphi}_{a b}: S_{\alpha} \rightarrow S_{\alpha} \varphi_{\alpha}\left(z_{a}-z_{b}\right), \quad \hat{f}_{a b}: S_{\alpha} \rightarrow S_{\alpha} f_{\alpha}\left(z_{a}-z_{b}\right) .
$$

In the following we also use $\hat{E}_{1}: S_{\alpha} \rightarrow S_{\alpha} E_{1}\left(\omega_{\alpha}\right)$. Note that

$$
\hat{\varphi}_{a b}^{*}=-\hat{\varphi}_{b a}
$$

in the sense that $\left\langle S^{a} \hat{\varphi}_{a b}\left(S^{b}\right)\right\rangle=-\left\langle S^{b} \hat{\varphi}_{b a}\left(S^{a}\right)\right\rangle$ due to (A.6). Similarly, $\hat{f}_{a b}^{*}=\hat{f}_{b a}, \hat{\wp}^{*}=\hat{\wp}$ and $\hat{E}_{1}^{*}=-\hat{E}_{1}$.

The commutativity of the Hamiltonians with respect to (2.1) follows from the underlying linear $r$-matrix structure of the Belavin-Drinfel'd type: $r_{12}^{\mathrm{BD}}(z, w)=\sum_{\alpha \in \Gamma^{\prime}} \varphi_{\alpha}(z-w) T_{\alpha} \otimes T_{-\alpha}[8]$. Note also that the Hamiltonians $H_{1, a}$ are not independent:

$$
\sum_{a=1}^{n} H_{1, a}=-\frac{1}{N} \sum_{a=1}^{n} \sum_{c \neq a}\left\langle S^{a} \hat{\varphi}_{a c}\left(S^{c}\right)\right\rangle \stackrel{(2.5)}{=} 0 .
$$

The appropriate number of independent Hamiltonians is achieved by taking into account $H_{0}$ and all higher Hamiltonians.

Let us write down equations of motion with respect to the Hamiltonians (2.3), (2.4):

$$
\begin{aligned}
& \partial_{t_{a}} S^{a}=\left\{H_{1, a}, S^{a}\right\}=-\sum_{c \neq a}\left[S^{a}, \hat{\varphi}_{a c}\left(S^{c}\right)\right], \\
& \partial_{t_{a}} S^{b}=\left\{H_{1, a}, S^{b}\right\}=\left[S^{b}, \hat{\varphi}_{b a}\left(S^{a}\right)\right], \\
& \partial_{t_{0}} S^{a}=\left\{H_{0}, S^{a}\right\}=\left[S^{a}, \hat{\wp}\left(S^{a}\right)\right]-\sum_{c \neq a}\left[S^{a}, \hat{f}_{a c}\left(S^{c}\right)\right] .
\end{aligned}
$$

\footnotetext{
${ }^{4}$ Note that we use both the Eisenstein and the Weierstrass functions. They are simply related (A.3), (A.4).
} 
Proposition 2.1. The equations of motion (2.6)-(2.8) can be presented in the Lax form (1.6) with the Lax matrix $L(z)$ defined in (2.2) and $M$-matrices given as follows:

$$
\begin{aligned}
& M_{a}=\sum_{\alpha \in \Gamma_{N}^{\prime}} S_{\alpha}^{a} T_{\alpha} \varphi_{\alpha}\left(z-z_{a}\right), \\
& M_{0}=-\sum_{b=1}^{n} \sum_{\gamma \in \Gamma_{N}^{\prime}} S_{\gamma}^{b} T_{\gamma} f_{\gamma}\left(z-z_{b}\right) .
\end{aligned}
$$

Proof. The proof is direct. It is based on the usage of (B.16)-(B.20).

Let us prove identity (B.20) which is the most nontrivial here. For a generic point $w \in \Sigma_{\tau}$ consider $m_{\gamma}^{a}(z, w)=\varphi_{\gamma}(z-w) \varphi_{\gamma}\left(w-z_{a}\right)$ :

$$
\begin{aligned}
m_{\gamma}^{a} \stackrel{\stackrel{\text { B.19) }}{=}}{=} \varphi_{\gamma}\left(z-z_{a}\right)\left(E_{1}(z-w)+E_{1}\left(w-z_{a}\right)+E_{1}\left(\omega_{\gamma}\right)-E_{1}\left(\omega_{\gamma}+z-z_{a}\right)\right) \\
\quad=\varphi_{\gamma}\left(z-z_{a}\right)\left(E_{1}(z-w)+E_{1}\left(w-z_{a}\right)\right)-f_{\gamma}\left(z-z_{a}\right) .
\end{aligned}
$$

Combining (B.16) and (B.19) which are implied to be known we have:

$$
\begin{aligned}
& \varphi_{\beta}\left(z-z_{c}\right) m_{\gamma}^{a}=\left(\varphi_{\beta}\left(z-z_{c}\right) \varphi_{\gamma}(z-w)\right) \varphi_{\gamma}\left(w-z_{a}\right) \\
& \quad \stackrel{\text { B.16) }}{=} \varphi_{\beta}\left(w-z_{c}\right)\left(\varphi_{\beta+\gamma}(z-w) \varphi_{\gamma}\left(w-z_{a}\right)\right)+\varphi_{\beta+\gamma}\left(z-z_{c}\right)\left(\varphi_{\gamma}\left(z_{c}-w\right) \varphi_{\gamma}\left(w-z_{a}\right)\right) \\
& \quad \stackrel{\text { B.16), }(\mathrm{B} .19)}{=} \varphi_{\beta}\left(w-z_{c}\right) \varphi_{\beta+\gamma}\left(z-z_{a}\right) \varphi_{-\beta}\left(w-z_{a}\right)+\varphi_{\beta}\left(w-z_{c}\right) \varphi_{\gamma}\left(z-z_{a}\right) \varphi_{\beta}(z-w) \\
& \quad+\varphi_{\beta+\gamma}\left(z-z_{c}\right) \varphi_{\gamma}\left(z_{c}-z_{a}\right)\left(E_{1}\left(z_{c}-w\right)+E_{1}\left(w-z_{a}\right)+E_{1}\left(\omega_{\gamma}\right)-E_{1}\left(\omega_{\gamma}+z_{c}-z_{a}\right)\right) \\
& \quad \stackrel{(\mathrm{B} .19)}{=} \varphi_{\gamma}\left(z-z_{a}\right) m_{\beta}^{c}-\varphi_{\beta+\gamma}\left(z-z_{a}\right) \varphi_{\beta}\left(z_{a}-z_{c}\right)\left(E_{1}\left(w-z_{c}\right)+E_{1}\left(z_{a}-w\right)+E_{1}\left(\omega_{\beta}\right)\right. \\
& \left.\quad-E_{1}\left(\omega_{\beta}+z_{a}-z_{c}\right)\right)+\varphi_{\beta+\gamma}\left(z-z_{c}\right) \varphi_{\gamma}\left(z_{c}-z_{a}\right)\left(E_{1}\left(z_{c}-w\right)+E_{1}\left(w-z_{a}\right)+E_{1}\left(\omega_{\gamma}\right)\right. \\
& \left.\quad-E_{1}\left(\omega_{\gamma}+z_{c}-z_{a}\right)\right) \\
& =\varphi_{\gamma}\left(z-z_{a}\right) m_{\beta}^{c}-\varphi_{\beta+\gamma}\left(z-z_{c}\right) f_{\gamma}\left(z_{c}-z_{a}\right)+\varphi_{\beta+\gamma}\left(z-z_{a}\right) f_{\beta}\left(z_{a}-z_{c}\right) \\
& \quad+\left(E_{1}\left(z_{c}-w\right)+E_{1}\left(w-z_{a}\right)\right)\left(\varphi_{\beta+\gamma}\left(z-z_{c}\right) \varphi_{\gamma}\left(z_{c}-z_{a}\right)+\varphi_{\beta+\gamma}\left(z-z_{a}\right) \varphi_{\beta}\left(z_{a}-z_{c}\right)\right) \\
& \quad \stackrel{\mathrm{B} .16)}{=} \varphi_{\gamma}\left(z-z_{a}\right) m_{\beta}^{c}-\varphi_{\beta+\gamma}\left(z-z_{c}\right) f_{\gamma}\left(z_{c}-z_{a}\right)+\varphi_{\beta+\gamma}\left(z-z_{a}\right) f_{\beta}\left(z_{a}-z_{c}\right) \\
& \quad+\left(E_{1}\left(z_{c}-w\right)+E_{1}\left(w-z_{a}\right)\right) \varphi_{\gamma}\left(z-z_{a}\right) \varphi_{\beta}\left(z-z_{c}\right) .
\end{aligned}
$$

This ends the proof of (B.20).

\subsection{Useful reformulation}

In this subsection we rewrite the equations of motion in a form which will be convenient for $1+1$ generalization. First, consider the following expressions for $a=1, \ldots, n$ :

$$
\begin{aligned}
& \sum_{\gamma \in \Gamma_{N}^{\prime}} T_{\gamma} \varphi_{\gamma}\left(z-z_{a}\right) \sum_{c \neq a} S_{\gamma}^{c} \varphi_{\gamma}\left(z_{a}-z_{c}\right) \\
& \quad \stackrel{(\mathrm{B} .19)}{=} \sum_{\gamma \in \Gamma_{N}^{\prime}} T_{\gamma} \sum_{c \neq a} S_{\gamma}^{c} \varphi_{\gamma}\left(z-z_{c}\right)\left(E_{1}\left(z-z_{a}\right)+E_{1}\left(z_{a}-z_{c}\right)+E_{1}\left(\omega_{\gamma}\right)-E_{1}\left(z-z_{c}+\omega_{\gamma}\right)\right) \\
& \quad=E_{1}\left(z-z_{a}\right)\left(L-M_{a}\right)+\sum_{c \neq a} M_{c} E_{1}\left(z_{a}-z_{c}\right)+M_{0}+\sum_{\gamma \in \Gamma_{N}^{\prime}} T_{\gamma} S_{\gamma}^{a} f_{\gamma}\left(z-z_{a}\right) \\
& \quad=E_{1}\left(z-z_{a}\right) L+\sum_{c \neq a} M_{c} E_{1}\left(z_{a}-z_{c}\right)+M_{0}-\sum_{\gamma \in \Gamma_{N}^{\prime}} T_{\gamma} S_{\gamma}^{a} F_{\gamma}\left(z-z_{a}\right) .
\end{aligned}
$$


Then let us define new $M$-matrices in the following way:

$$
\tilde{M}_{a}=\sum_{\gamma \in \Gamma_{N}^{\prime}} T_{\gamma} S_{\gamma}^{a} F_{\gamma}\left(z-z_{a}\right)+\sum_{\gamma \in \Gamma_{N}^{\prime}} T_{\gamma} \eta_{\gamma}^{\prime a} \varphi_{\gamma}\left(z-z_{a}\right), \quad a=1, \ldots, n
$$

where

$$
\eta^{\prime a}=\sum_{c \neq a} T_{\gamma} S_{\gamma}^{c} \varphi_{\gamma}\left(z_{a}-z_{c}\right)=\sum_{c \neq a} M^{c}\left(z_{a}\right)=\operatorname{Res}_{z=z_{a}}\left(\frac{1}{z-z_{a}} L(z)\right) .
$$

From (2.11) we can see that the new $M$-matrices are the linear combinations of (2.9), (2.10):

$$
\tilde{M}_{a}=E_{1}\left(z-z_{a}\right) L+\sum_{c \neq a} M_{c} E_{1}\left(z_{a}-z_{c}\right)+M_{0} .
$$

Then the Lax equations yield

$$
\partial_{\tilde{t}_{a}} L=\left[L, \sum_{c \neq a} M_{c} E_{1}\left(z_{a}-z_{c}\right)+M_{0}\right]=\sum_{c \neq a} E_{1}\left(z_{a}-z_{c}\right) \partial_{t_{c}} L+\partial_{t_{0}} L
$$

and the equations of motion are:

$$
\begin{aligned}
\partial_{\tilde{t}_{a}} S^{a} & =\sum_{c \neq a} E_{1}\left(z_{a}-z_{c}\right) \partial_{t_{c}} S^{a}+\partial_{t_{0}} S^{a} \\
& \stackrel{(2.6)-(2.8)}{=} \sum_{c \neq a}\left[S^{a}, E_{1}\left(z_{a}-z_{c}\right) \hat{\varphi}_{a c}\left(S^{c}\right)-\hat{f}_{a c}\left(S^{c}\right)\right]+\left[S^{a}, \hat{\wp}\left(S^{a}\right)\right]
\end{aligned}
$$

while for $b \neq a$ :

$$
\begin{aligned}
\partial_{\tilde{t}_{a}} S^{b}= & \partial_{t_{b}} S^{b} E_{1}\left(z_{a}-z_{b}\right)+\sum_{c \neq a, b} \partial_{t_{c}} S^{b} E_{1}\left(z_{a}-z_{c}\right)+\partial_{t_{0}} S^{b}=\left[S^{b}, \hat{\wp} S^{b}\right] \\
& -E_{1}\left(z_{a}-z_{b}\right) \sum_{c \neq b}\left[S^{b}, \hat{\varphi}_{b c}\left(S^{c}\right)\right]+\sum_{c \neq a, b} E_{1}\left(z_{a}-z_{c}\right)\left[S^{b}, \hat{\varphi}_{b c}\left(S^{c}\right)\right]-\sum_{c \neq b}\left[S^{b}, \hat{f}_{b c}\left(S^{c}\right)\right] \\
& =\left[S^{b}, \hat{\wp}\left(S^{b}\right)+E_{1}\left(z_{b}-z_{a}\right) \hat{\varphi}_{b a}\left(S^{a}\right)-\hat{f}_{b a}\left(S^{a}\right)\right] \\
& +\sum_{c \neq a, b}\left[S^{b},\left(E_{1}\left(z_{b}-z_{a}\right)+E_{1}\left(z_{a}-z_{c}\right)\right) \hat{\varphi}_{b c}\left(S^{c}\right)-\hat{f}_{b c}\left(S^{c}\right)\right] .
\end{aligned}
$$

Finally, we have

$$
\begin{aligned}
\partial_{\tilde{t}_{a}} S^{a} & =\left[S^{a}, \hat{\wp} S^{a}\right]+\sum_{c \neq a}\left[S^{a}, \hat{F}_{a c}\left(S^{c}\right)\right] \\
\partial_{\tilde{t}_{a}} S^{b} & =\left[S^{b}, \hat{\wp} S^{b}\right]+\left[S^{b}, \hat{F}_{b a}\left(S^{a}\right)\right]+\sum_{c \neq a, b}\left[S^{b}, \hat{\varphi}_{b a}\left(\hat{\varphi}_{a c}\left(S^{c}\right)\right)\right] \\
& =\sum_{c \neq a}\left[S^{b}, \hat{\varphi}_{b a}\left(\hat{\varphi}_{a c}\left(S^{c}\right)\right)\right]+\left[S^{b}, \hat{F}_{b a}\left(S^{a}\right)\right]=\left[S^{b}, \hat{\varphi}_{b a}\left(\eta^{\prime a}\right)\right]+\left[S^{b}, \hat{F}_{b a}\left(S^{a}\right)\right] .
\end{aligned}
$$

The corresponding Hamiltonians are obtained in the same way:

$$
H_{0}+\sum_{c \neq a} E_{1}\left(z_{a}-z_{c}\right) H_{c}=\frac{1}{2 N} \sum_{c}\left\langle S^{c} \hat{\wp} S^{c}\right\rangle-\frac{1}{2 N} \sum_{b \neq c}\left\langle S^{c} \hat{f}_{c b}\left(S^{b}\right)\right\rangle
$$




$$
\begin{aligned}
& -\frac{1}{N} \sum_{c \neq a} E_{1}\left(z_{a}-z_{c}\right) \sum_{b \neq c}\left\langle S^{c} \hat{\varphi}_{c b}\left(S^{b}\right)\right\rangle=\frac{1}{2 N} \sum_{c}\left\langle S^{c} \hat{\wp} S^{c}\right\rangle-\frac{1}{2 N} \sum_{b, c \neq a, b \neq c}\left\langle S^{c} \hat{f}_{c b}\left(S^{b}\right)\right\rangle \\
& -\frac{1}{N} \sum_{c \neq a}\left\langle S^{a} \hat{f}_{a c}\left(S^{c}\right)\right\rangle-\frac{1}{2 N} \sum_{b, c \neq a, b \neq c}\left(E_{1}\left(z_{a}-z_{c}\right)-E_{1}\left(z_{a}-z_{b}\right)\right)\left\langle S^{c} \hat{\varphi}_{c b}\left(S^{b}\right)\right\rangle \\
& -\frac{1}{N} \sum_{c \neq a} E_{1}\left(z_{a}-z_{c}\right)\left\langle S^{c} \hat{\varphi}_{c a}\left(S^{a}\right)\right\rangle \\
& =\frac{1}{2 N} \sum_{c}\left\langle S^{c} \hat{\wp} S^{c}\right\rangle+\frac{1}{N} \sum_{c \neq a}\left\langle S^{a} \hat{F}_{a c}\left(S^{c}\right)\right\rangle+\frac{1}{2 N} \sum_{b, c \neq a, b \neq c}\left\langle S^{c} \hat{\varphi}_{c a}\left(\hat{\varphi}_{a b}\left(S^{b}\right)\right)\right\rangle .
\end{aligned}
$$

The last one term equals:

$$
\begin{gathered}
\frac{1}{2 N} \sum_{b, c \neq a, b \neq c}\left\langle S^{c} \hat{\varphi}_{c a}\left(\hat{\varphi}_{a b}\left(S^{b}\right)\right)\right\rangle=\frac{1}{2 N} \sum_{b, c \neq a}\left\langle S^{c} \hat{\varphi}_{c a}\left(\hat{\varphi}_{a b}\left(S^{b}\right)\right)\right\rangle-\frac{1}{2 N} \sum_{c \neq a}\left\langle S^{c} \hat{\varphi}_{c a}\left(\hat{\varphi}_{a c}\left(S^{c}\right)\right)\right\rangle \\
=\frac{1}{2 N} \sum_{b, c \neq a}\left\langle S^{c} \hat{\varphi}_{c a}\left(\hat{\varphi}_{a b}\left(S^{b}\right)\right)\right\rangle-\frac{1}{2 N} \sum_{c \neq a}\left\langle S^{c} \hat{\wp}\left(S^{c}\right)\right\rangle+\frac{1}{2 N} \sum_{c \neq a}\left\langle S^{c} S^{c}\right\rangle \wp\left(z_{a}-z_{c}\right) .
\end{gathered}
$$

From $(2.13),(2.14)$ we conclude that the Hamiltonians for the reformulated version of the Gaudin model are of the form:

$$
\tilde{H}_{a}=\frac{1}{2 N}\left\langle S^{a} \hat{\wp} S^{a}\right\rangle+\frac{1}{N} \sum_{c \neq a}\left\langle S^{a} \hat{F}_{a c}\left(S^{c}\right)\right\rangle+\frac{1}{2 N} \sum_{b, c \neq a}\left\langle S^{c} \hat{\varphi}_{c a}\left(\hat{\varphi}_{a b}\left(S^{b}\right)\right)\right\rangle, \quad a=1, \ldots, n
$$

or

$$
\tilde{H}_{a}=\frac{1}{2 N} \sum_{c}\left\langle S^{c} \hat{\wp} S^{c}\right\rangle+\frac{1}{N} \sum_{c \neq a}\left\langle S^{a} \hat{F}_{a c}\left(S^{c}\right)\right\rangle+\frac{1}{2 N} \sum_{\substack{b, c \neq a, b \neq c}}\left\langle S^{c} \hat{\varphi}_{c a}\left(\hat{\varphi}_{a b}\left(S^{b}\right)\right)\right\rangle, \quad a=1, \ldots, n .
$$

Two last forms of the Hamiltonians are differ by the constant $\frac{1}{2 N} \sum_{c \neq a}\left\langle S^{c} S^{c}\right\rangle \wp\left(z_{a}-z_{c}\right)$. Let us summarize the obtained in results in

Proposition 2.2. The dynamics of the Gaudin model produced by Hamiltonians

$$
\tilde{H}_{a}=\frac{1}{2 N}\left\langle S^{a} \hat{\wp} S^{a}\right\rangle+\frac{1}{N} \sum_{c \neq a}\left\langle S^{a} \hat{F}_{a c}\left(S^{c}\right)\right\rangle+\frac{1}{2 N} \sum_{b, c \neq a}\left\langle S^{c} \hat{\varphi}_{c a}\left(\hat{\varphi}_{a b}\left(S^{b}\right)\right)\right\rangle
$$

is given by equations

$$
\begin{aligned}
& \partial_{\tilde{t}_{a}} S^{a}=\left[S^{a}, \hat{\wp} S^{a}\right]+\sum_{c \neq a}\left[S^{a}, \hat{F}_{a c}\left(S^{c}\right)\right], \\
& \partial_{\tilde{t}_{a}} S^{b}=\left[S^{b}, \hat{\varphi}_{b a}\left(\eta^{\prime a}\right)\right]+\left[S^{b}, \hat{F}_{b a}\left(S^{a}\right)\right], \quad \eta^{\prime a}=\sum_{c \neq a} \hat{\varphi}_{a c}\left(S^{c}\right)
\end{aligned}
$$

and can be presented in the Lax form with $L(z)$ from (2.2) and

$$
\tilde{M}_{a}=\sum_{\gamma \in \Gamma_{N}^{\prime}} T_{\gamma} S_{\gamma}^{a} F_{\gamma}\left(z-z_{a}\right)+\sum_{\gamma \in \Gamma_{N}^{\prime}} T_{\gamma} \eta_{\gamma}^{\prime a} \varphi_{\gamma}\left(z-z_{a}\right), \quad a=1, \ldots, n .
$$

The Gaudin Hamiltonians (2.3) and (2.15) are simplified when written in terms of $\eta^{\prime a}(2.12)$ :

$$
H_{a}=-\left\langle S^{a} \eta^{\prime a}\right\rangle, \quad \tilde{H}_{a}=\frac{1}{2 N}\left\langle S^{a} \hat{\wp} S^{a}\right\rangle-\frac{1}{2 N}\left\langle\left(\eta^{\prime a}\right)^{2}\right\rangle+\frac{1}{N} \sum_{c \neq a}\left\langle S^{a} \hat{F}_{a c}\left(S^{c}\right)\right\rangle .
$$


In the end of the section let us also give the rational "reformulated" version since it is more illuminating:

$$
\tilde{M}_{a}=\sum_{c \neq a} \frac{M_{c}}{z_{a}-z_{c}}+\frac{1}{z-z_{a}} L=\frac{1}{z-z_{a}} M_{a}+\frac{\eta^{\prime a}}{z-z_{a}},
$$

where

$$
\eta^{\prime a}=\sum_{c \neq a} \frac{S^{c}}{z_{a}-z_{c}} .
$$

Hamiltonians:

$$
\tilde{H}_{a}=-\frac{1}{2 N}\left\langle\left(\sum_{c \neq a} \frac{S^{c}}{z_{a}-z_{c}}\right)^{2}\right\rangle+\frac{1}{N} \sum_{c \neq a} \frac{\left\langle S^{a} S^{c}\right\rangle}{\left(z_{a}-z_{c}\right)^{2}} .
$$

The later follows from simple evaluation:

$$
\begin{aligned}
& \sum_{c \neq a} \frac{H_{c}}{z_{a}-z_{c}}=-\frac{1}{N} \sum_{c \neq a} \sum_{b \neq c} \frac{1}{z_{a}-z_{c}} \frac{\left\langle S^{c} S^{b}\right\rangle}{z_{c}-z_{b}}=\frac{1}{N} \sum_{c \neq a} \frac{\left\langle S^{a} S^{c}\right\rangle}{\left(z_{a}-z_{c}\right)^{2}} \\
& +\frac{1}{N} \sum_{b, c \neq a ; b \neq c} \frac{\left\langle S^{c} S^{b}\right\rangle}{\left(z_{c}-z_{a}\right)\left(z_{c}-z_{b}\right)}=-\frac{1}{2 N}\left\langle\left(\eta^{\prime a}\right)^{2}\right\rangle+\frac{1}{N} \sum_{c \neq a} \frac{\left\langle S^{a} S^{c}\right\rangle}{\left(z_{a}-z_{c}\right)^{2}}+\frac{1}{2 N} \sum_{c \neq a} \frac{\left\langle\left(S^{c}\right)^{2}\right\rangle}{\left(z_{a}-z_{c}\right)^{2}} .
\end{aligned}
$$

The last one term is the analogue of the constant $\frac{1}{2 N} \sum_{c \neq a}\left\langle S^{c} S^{c}\right\rangle \wp\left(z_{a}-z_{c}\right)$ in (2.14). The corresponding equations of motion are:

$$
\begin{aligned}
& \partial_{\tilde{t}_{a}} S^{a}=\sum_{c \neq a} \frac{\left[S^{a}, S^{c}\right]}{\left(z_{a}-z_{c}\right)^{2}}, \\
& \partial_{\tilde{t}_{a}} S^{b}=\frac{\left[S^{b}, S^{a}\right]}{\left(z_{a}-z_{b}\right)^{2}}+\frac{1}{z_{a}-z_{b}} \sum_{c \neq a} \frac{\left[S^{c}, S^{b}\right]}{z_{a}-z_{c}}=\frac{\left[S^{b}, S^{a}\right]}{\left(z_{a}-z_{b}\right)^{2}}+\frac{\left[\eta^{a}, S^{b}\right]}{z_{a}-z_{b}} .
\end{aligned}
$$

\section{Field version}

\section{$3.1 \quad 1+1 \operatorname{sl}(N, \mathbb{C})$ Gaudin model}

The general construction of the field version for the Hitchin systems was described in [19]. For our current purposes we only need to define the phase space. By analogy with mechanics let us consider a collection (direct product) of $n$ orbits assigned to the marked points, i.e. let $\operatorname{Res}_{z=z_{a}} L(z)=S^{a}(x)$ be elements of the loop coalgebras $\hat{\mathrm{sl}}^{*}(N, \mathbb{C})$ and $x$ be a loop variable. We imply that the values of the invariants under the coadjoint action (or the eigenvalues of $S^{a}$ ) are fixed. More over we assume for simplicity that the eigenvalues are $\mathbb{C}$-numbers (independent of $x$ ). From the physical point of view it means that the magnetic momentum vector is normalized (as it is assumed in the Landau-Lifshitz model). The boundary conditions are chosen to be periodic. In summary, $S^{a}(x)$ are $\hat{\mathrm{sl}}^{*}(N, \mathbb{C})$-valued periodic functions on a unit circle $\mathbb{S}^{1}: S^{a}(x+2 \pi)=S^{a}(x)$ with eigenvalues $\left\{\lambda_{k, a}, k=1, \ldots, N, a=1, \ldots, n\right\}$ fixed to be $\mathbb{C}$-numbers: $\partial_{x} \lambda_{a}=0$.

In the field case the Lax equations (1.6) a replaced by the zero-curvature equations:

$$
\partial_{t_{a}} L-k \partial_{x} M_{a}=\left[L, M_{a}\right]
$$


In fact, the numeration of $M_{a}$ should include two type of indices as in (2.3): the first one type describes the number of the flow in the hierarchy and runs over $1, \ldots, N$ in $0+1$ mechanics or $1, \ldots, \infty$ in $1+1$ field theory while the second one runs over $1, \ldots, n$ in both cases and describes the assignment of the Hamiltonians to the marked points. In this paper we are not going to concern the whole hierarchy but only two first flows (as we did in $0+1$ case).

We will see that the first $n$ flows of the hierarchy corresponds to the Gaudin Hamiltonians in the standard description (2.3) supplemented by the momenta $P_{a}$ along $x$ while the second $n$ flows naturally related to reformulated version (2.15)

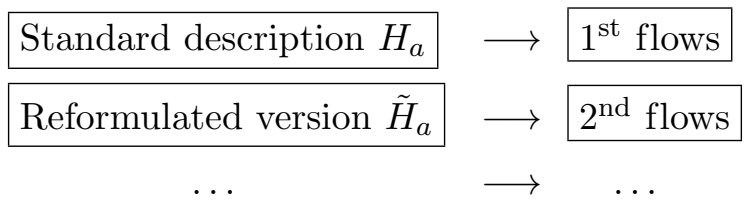

Thus we do not use multi-index for times. It is sufficient to use $t_{a}$ and $\tilde{t}_{a}$ for our purposes and we keep these notations for the field version.

It should be mentioned that the field generalization of the Lax pair into "L-A" pair satisfying (3.1) is nontrivial. The fact that the $L$-matrix (2.2) is unchanged in the field version follows from the triviality of the moduli space of bundles of degree one. It is explained in [19] in detail. As a result we deal with the following Lax matrix:

$$
L=\sum_{c=1}^{n} \sum_{\gamma \in \Gamma_{N}^{\prime}} T_{\gamma} S_{\gamma}^{c} \varphi_{\gamma}\left(z-z_{c}\right) .
$$

The $M_{a}$-matrices for the first flow coincide with the mechanical versions either:

$$
M_{a}=\sum_{\gamma \in \Gamma_{N}^{\prime}} T_{\gamma} S_{\gamma}^{a} \varphi_{\gamma}\left(z-z_{a}\right) .
$$

Proposition 3.1. The zero-curvature equations (3.1) with $L$ from (3.2) and $M_{a}$ from (3.3) are equivalent to the following equations:

$$
\begin{aligned}
& \partial_{t_{a}} S^{a}-k \partial_{x} S^{a}=-\sum_{c \neq a}\left[S^{a}, \hat{\varphi}_{a c}\left(S^{c}\right)\right], \\
& \partial_{t_{a}} S^{b}=\left[S^{b}, \hat{\varphi}_{b a}\left(S^{a}\right)\right] .
\end{aligned}
$$

The proof is the same as in the $0+1$ case. As we will see below the Hamiltonian corresponding to $M_{a}$ has the form

$$
\mathcal{H}_{a}=\oint_{\mathbb{S}^{1}} \mathrm{~d} x\left(P_{a}+H_{a}(S(x))\right),
$$

where $\oint_{\mathbb{S}^{1}} \mathrm{~d} x P_{a}$ is the shift operator in the loop algebra $\hat{\operatorname{sl}}(N, \mathbb{C}):\left\{\oint_{\mathbb{S}^{1}} \mathrm{~d} x P_{a}(x), S^{b}(y)\right\}=$ $\delta_{a b} \partial_{y} S^{b}(y)$ and $H_{a}$ is defined as in (1.5) or (2.3). Thus the Hamiltonian describing equations (3.4) has the form:

$$
\mathcal{H}_{a}=\oint_{\mathbb{S}^{1}} \mathrm{~d} x\left(P_{a}-\frac{1}{N} \sum_{c \neq a}\left\langle S^{a} \hat{\varphi}_{a c}\left(S^{c}\right)\right\rangle\right) .
$$

The phase space is a direct product of the symplectic orbits of the loop group $\hat{\mathrm{SL}}(N, \mathbb{C})$ with linear Poisson structure:

$$
\left\{S_{\alpha}^{a}(x), S_{\beta}^{b}(y)\right\}=\delta_{a b} \delta(x-y) c_{\alpha, \beta} S_{\alpha+\beta}^{a}(x), \quad a, b=1, \ldots, n .
$$

The second flows are of our main interest. 
Proposition 3.2. The zero-curvature equations

$$
\partial_{\tilde{t}_{a}} L-k \partial_{x} \tilde{M}_{a}=\left[L, \tilde{M}_{a}\right]
$$

with $L$ from (3.2) and

$$
\tilde{M}_{a}=\sum_{\gamma \in \Gamma_{N}^{\prime}} T_{\gamma} S_{\gamma}^{a} F_{\gamma}\left(z-z_{a}\right)+\sum_{\gamma \in \Gamma_{N}^{\prime}} T_{\gamma} \eta_{\gamma}^{a} \varphi_{\gamma}\left(z-z_{a}\right), \quad a=1, \ldots, n,
$$

where $\eta^{a}=\eta^{\prime a}+\Delta \eta^{a}, \eta^{\prime a}=\sum_{c \neq a} \hat{\varphi}_{a c}\left(S^{c}\right)$ are equivalent to the following equations:

$$
\begin{aligned}
& \partial_{\tilde{t}_{a}} S^{a}-k \partial_{x} \eta^{a}=\left[S^{a}, \hat{\wp}\left(S^{a}\right)\right]+\sum_{c \neq a}\left[S^{a}, \hat{F}_{a c}\left(S^{c}\right)\right]+\sum_{c \neq a}\left[\hat{\varphi}_{a c}\left(S^{c}\right), \eta^{a}\right] \\
& +\left[\hat{E}_{1}\left(S^{a}\right), \Delta \eta^{a}\right]+\left[S^{a}, \hat{E}_{1}\left(\Delta \eta^{a}\right)\right]-\hat{E}_{1}\left[S^{a}, \Delta \eta^{a}\right], \\
& \partial_{\tilde{t}_{a}} S^{b}=\left[S^{b}, \hat{\varphi}_{b a}\left(\eta^{a}\right)\right]+\left[S^{b}, \hat{F}_{b a}\left(S^{a}\right)\right] \text {, } \\
& -k \partial_{x} S^{a}=\left[S^{a}, \Delta \eta^{a}\right] .
\end{aligned}
$$

The proof is also similar to the one given for the $0+1$ case. Functions $\eta^{a}$ are not uniquely defined by equations $-k \partial_{x} S^{a}=\left[S^{a}, \Delta \eta^{a}\right]$. We fix this ambiguity by requiring $\eta^{a} \rightarrow \eta^{a}=$ $\sum_{c \neq a} \hat{\varphi}_{a c}\left(S^{c}\right)$ or $\Delta \eta^{a} \rightarrow 0$ in $0+1$ limit. As for the equation $-k \partial_{x} S^{a}=\left[S^{a}, \Delta \eta^{a}\right]$ itself only some special cases were studied such as "vector" case [33, 34] and "Grassmannian" case (special coadjoint orbits) [35]. For $\hat{\mathrm{sl}}(2, \mathbb{C})$ case the answer is well known: $\Delta \eta^{a}=-\frac{k}{4 \lambda_{a}^{2}}\left[S^{a}, S_{x}^{a}\right]$.

\subsection{2-site case and principal chiral model}

L-A pair for the principal chiral model was suggested in [29] (see also [23, 30, 36, 37]). Consider the first flows of the Gaudin model (3.4) with 2 sites or marked points $(n=2)$. It is convenient to start from the rational version:

$$
L=\frac{S^{1}}{z-z_{1}}+\frac{S^{2}}{z-z_{2}}=M_{1}+M_{2}
$$

The corresponding $M$-matrix is known to be

$$
M=M_{1}-M_{2}=\frac{S^{1}}{z-z_{1}}-\frac{S^{2}}{z-z_{2}} .
$$

Therefore the equations of motion are

$$
\begin{aligned}
\partial_{t} S^{1}-k \partial_{x} S^{1} & =-\frac{2}{z_{1}-z_{2}}\left[S^{1}, S^{2}\right], \\
\partial_{t} S^{2}+k \partial_{x} S^{2} & =\frac{2}{z_{1}-z_{2}}\left[S^{1}, S^{2}\right] .
\end{aligned}
$$

Then the Hamiltonian describing equations (3.7) has a form ${ }^{5}$ :

$$
\mathcal{H}=\mathcal{H}_{1}-\mathcal{H}_{2}=\oint_{\mathbb{S}^{1}} \mathrm{~d} x\left(P_{1}-P_{2}-\frac{\left\langle S^{1} S^{2}\right\rangle}{z_{1}-z_{2}}\right)
$$

and the phase space is a direct product of two symplectic orbits of the loop group $\hat{\mathrm{SL}}(N, \mathbb{C})$ with the linear Poisson structure:

$$
\left\{S_{\alpha}^{a}(x), S_{\beta}^{b}(y)\right\}=\delta_{a b} \delta(x-y) c_{\alpha, \beta} S_{\alpha+\beta}^{a}(x), \quad a, b=1,2 .
$$

\footnotetext{
${ }^{5}$ See Section 4.3 and (4.24).
} 
Remark 3.1. One can make a substitution $S^{1}=\frac{1}{2}\left(l_{0}+l_{1}\right)$ and $S^{2}=\frac{1}{2}\left(l_{0}-l_{1}\right)$ to represent equations (3.7) in its traditional form

$$
\begin{aligned}
& \partial_{t} l_{1}-k \partial_{x} l_{0}+\frac{2}{z_{1}-z_{2}}\left[l_{1}, l_{0}\right]=0, \\
& \partial_{t} l_{0}-k \partial_{x} l_{1}=0
\end{aligned}
$$

or change the coordinates $(x, t)$ to "light-cone" coordinates $\xi=\frac{t+k^{-1} x}{2}, \eta=\frac{t-k^{-1} x}{2}$ :

$$
\begin{aligned}
\partial_{\eta} S^{1} & =-\frac{2}{z_{1}-z_{2}}\left[S^{1}, S^{2}\right], \\
\partial_{\xi} S^{2} & =\frac{2}{z_{1}-z_{2}}\left[S^{1}, S^{2}\right] .
\end{aligned}
$$

Elliptic case. For L-A pair $L=M_{1}+M_{2}$ and $M=M_{1}-M_{2}$ with $M_{a}=\sum_{\alpha \in \Gamma_{N}^{\prime}} T_{\alpha} S_{\alpha}^{a} \varphi_{\alpha}\left(z-z_{a}\right)$, $a=1,2$ the equations $(3.4)$ yields $\left(\partial_{t}=\partial_{t_{1}}-\partial_{t_{2}}\right)$ :

$$
\begin{aligned}
& \partial_{t} S^{1}-k \partial_{x} S^{1}=-2\left[S^{1}, \hat{\varphi}_{12}\left(S^{2}\right)\right], \\
& \partial_{t} S^{2}+k \partial_{x} S^{2}=2\left[S^{2}, \hat{\varphi}_{21}\left(S^{1}\right)\right] .
\end{aligned}
$$

or by analogy with $(3.8)$

$$
\begin{aligned}
& \partial_{\eta} S^{1}=-2\left[S^{1}, \hat{\varphi}_{12}\left(S^{2}\right)\right], \\
& \partial_{\xi} S^{2}=2\left[S^{2}, \hat{\varphi}_{21}\left(S^{1}\right)\right] .
\end{aligned}
$$

In $\operatorname{sl}(2, \mathbb{C})$ case this result was obtained by I. Cherednik [30]. Here we see that the principal chiral model corresponds to the special (2-site) case of the first flows of $1+1$ Gaudin model. It should be also mentioned that in [30] the equations for $\operatorname{sl}(2, \mathbb{C})$ case were obtained as a field version of XYZ model, i.e. from the second flow of 1-site Gaudin model (or sl(2, $\mathbb{C})$ elliptic top). It may be explained as follows: consider stationary solutions $S^{a}=S^{a}(\eta)$ (or $\partial_{\xi} S^{a}=0$ ). Then fixing the ambiguity in solutions of the equation $\left[S^{2}, \hat{\varphi}_{21}\left(S^{1}\right)\right]=0$ as $S^{2}=-\frac{1}{2} \hat{\varphi}_{21}\left(S^{1}\right)$ we have

$$
\partial_{\eta} S^{1}=\left[S^{1}, \hat{\varphi}_{12} \hat{\varphi}_{21}\left(S^{1}\right)\right]=\left[S^{1}, \hat{\wp}\left(S^{1}\right)\right],
$$

which is the equation of $\operatorname{sl}(N, \mathbb{C})$ elliptic top (or 1-site elliptic Gaudin model) corresponding to the second flow $H=\frac{1}{2 N}\left\langle S^{1} \hat{\wp}\left(S^{1}\right)\right\rangle$.

\section{$4 \operatorname{sl}(2, \mathbb{C}) 1+1$ Gaudin models}

\subsection{1+1 XXX Gaudin magnet: interacting Heisenberg models}

Let us consider the case $\operatorname{Res}_{z_{a}} L(z)=S^{a} \in \operatorname{sl}(2, \mathbb{C})$ in detail. The linear Poisson-Lie structure in this case:

$$
\left\{S_{\alpha}^{a}, S_{\beta}^{b}\right\}=2 \sqrt{-1} \delta^{a b} \varepsilon_{\alpha \beta \gamma} S_{\gamma}^{a},
$$

where $S_{\alpha}^{a}$ are coefficients in the basis of Pauli matrices: $S^{a}=\sum_{\alpha=1}^{3} S_{\alpha}^{a} \sigma_{\alpha}$.

The Gaudin Hamiltonians are:

$$
H_{a}=-\sum_{c \neq a} \frac{S_{1}^{a} S_{1}^{c}+S_{2}^{a} S_{2}^{c}+S_{3}^{a} S_{3}^{c}}{z_{a}-z_{c}}
$$


while the Hamiltonians of the reformulated version are

$$
\tilde{H}_{a}=\frac{1}{2} \sum_{c \neq a} \frac{\left\langle S^{a} S^{c}\right\rangle}{\left(z_{a}-z_{c}\right)^{2}}-\frac{1}{4}\left\langle\left(\sum_{c \neq a} \frac{S^{c}}{z_{a}-z_{c}}\right)^{2}\right\rangle+\frac{H_{a}^{2}}{2 \lambda_{a}^{2}} .
$$

Since

$$
\begin{aligned}
\sum_{c \neq a} & \frac{H_{c}}{z_{a}-z_{c}}+\frac{H_{a}^{2}}{2 \lambda_{a}^{2}}=-\frac{1}{2} \sum_{c \neq a} \sum_{b \neq c} \frac{1}{z_{a}-z_{c}} \frac{\left\langle S^{c} S^{b}\right\rangle}{z_{c}-z_{b}}+\frac{H_{a}^{2}}{2 \lambda_{a}^{2}} \\
= & \frac{1}{2} \sum_{c \neq a} \frac{\left\langle S^{a} S^{c}\right\rangle}{\left(z_{a}-z_{c}\right)^{2}}+\frac{1}{2} \sum_{b, c \neq a ; b \neq c} \frac{\left\langle S^{c} S^{b}\right\rangle}{\left(z_{c}-z_{a}\right)\left(z_{c}-z_{b}\right)}+\frac{H_{a}^{2}}{2 \lambda_{a}^{2}} \\
= & \frac{1}{2} \sum_{c \neq a} \frac{\left\langle S^{a} S^{c}\right\rangle}{\left(z_{a}-z_{c}\right)^{2}}-\frac{1}{4} \sum_{b, c \neq a ; b \neq c} \frac{\left\langle S^{c} S^{b}\right\rangle}{\left(z_{a}-z_{c}\right)\left(z_{a}-z_{b}\right)}+\frac{H_{a}^{2}}{2 \lambda_{a}^{2}} \\
= & \frac{1}{2} \sum_{c \neq a} \frac{\left\langle S^{a} S^{c}\right\rangle}{\left(z_{a}-z_{c}\right)^{2}}+\frac{1}{4} \sum_{c \neq a} \frac{\left\langle\left(S^{c}\right)^{2}\right\rangle}{\left(z_{a}-z_{c}\right)^{2}}-\frac{1}{4}\left\langle\left(\sum_{c \neq a} \frac{S^{c}}{z_{a}-z_{c}}\right)^{2}\right\rangle+\frac{H_{a}^{2}}{2 \lambda_{a}^{2}},
\end{aligned}
$$

then the corresponding equations of motion are:

$$
\begin{aligned}
& \partial_{\tilde{t}_{a}} S^{a}=\sum_{c \neq a} \frac{\left[S^{a}, S^{c}\right]}{\left(z_{a}-z_{c}\right)^{2}}-\frac{H_{a}}{\lambda_{a}^{2}} \sum_{c \neq a} \frac{\left[S^{a}, S^{c}\right]}{z_{a}-z_{c}} \\
& \partial_{\tilde{t}_{a}} S^{b}=\frac{\left[S^{b}, S^{a}\right]}{\left(z_{a}-z_{b}\right)^{2}}+\frac{1}{z_{a}-z_{b}} \sum_{c \neq a} \frac{\left[S^{c}, S^{b}\right]}{z_{a}-z_{c}}+\frac{H_{a}}{\lambda_{a}^{2}} \frac{\left[S^{a}, S^{b}\right]}{z_{a}-z_{b}}=\frac{\left[S^{b}, S^{a}\right]}{\left(z_{a}-z_{b}\right)^{2}}+\frac{\left[\eta^{\prime a}, S^{b}\right]}{z_{a}-z_{b}},
\end{aligned}
$$

where

$$
\eta^{\prime a}=\sum_{c \neq a} \frac{S^{c}}{z_{a}-z_{c}}+\frac{H_{a}}{\lambda_{a}^{2}} S^{a} .
$$

Remark 4.1. (4.4) differs from (2.16) by $\frac{H_{a}}{\lambda_{a}^{2}} S^{a}$ and the corresponding Hamiltonian (4.3) differs from (2.17) by $\frac{H_{a}^{2}}{2 \lambda_{a}^{2}}$. This difference does not follow from ansatz (3.5) but appears from the Hamiltonian description (see Section 4.3). The corresponding Lax pair is given by $L$ from (1.2) and

$$
\tilde{M}_{a}=\sum_{c \neq a} \frac{M_{c}}{z_{a}-z_{c}}+\frac{1}{z-z_{a}} L+\frac{H_{a}}{\lambda_{a}^{2}} M_{a}=\frac{1}{z-z_{a}} M_{a}+\frac{\eta^{\prime a}}{z-z_{a}} .
$$

$\mathbf{1}+\mathbf{1}$ version. Let $S^{a}(x) \in \widehat{\mathrm{sl}}(2, \mathbb{C})$ be periodic $\operatorname{sl}(2, \mathbb{C})$-valued functions on a circle $\mathbb{S}^{1}$ : $S^{a}(x+2 \pi)=S^{a}(x)$ with eigenvalues $\left\{\lambda_{a}\right\}$ fixed to be $\mathbb{C}$-numbers: $\partial_{x} \lambda_{a}=0$. The Poisson structure now is

$$
\left\{S_{\alpha}^{a}(x), S_{\beta}^{b}(y)\right\}=2 \sqrt{-1} \delta^{a b} \varepsilon_{\alpha \beta \gamma} S_{\gamma}^{a}(x) \delta(x-y) .
$$

Consider

$$
\tilde{M}_{a}=\frac{S^{a}}{\left(z-z_{a}\right)^{2}}+\frac{\eta^{a}}{z-z_{a}},
$$

where

$$
\eta^{a}=-\frac{k}{4 \lambda_{a}^{2}}\left[S^{a}, S_{x}^{a}\right]+\sum_{c \neq a} \frac{S^{c}}{z_{a}-z_{c}}+\frac{H_{a}}{\lambda_{a}^{2}} S^{a}=-\frac{k}{4 \lambda_{a}^{2}}\left[S^{a}, S_{x}^{a}\right]+\eta^{\prime a}, \quad S_{x}^{a} \equiv \partial_{x} S^{a} .
$$


Let us remark here that in $(0+1)$ limit $\eta^{a}=\eta^{\prime a}(4.4)$. Then the zero-curvature equation

$$
\partial_{\tilde{t}_{a}} L-k \partial_{x} \tilde{M}_{a}=\left[L, \tilde{M}_{a}\right]
$$

reads as follows

$$
\begin{aligned}
& \partial_{\tilde{t}_{a}} S^{a}-k \partial_{x} \eta^{a}=\left[\sum_{c \neq a} \frac{S^{c}}{z_{a}-z_{c}}, \eta^{a}\right]+\sum_{c \neq a} \frac{\left[S^{a}, S^{c}\right]}{\left(z_{a}-z_{c}\right)^{2}}, \\
& \partial_{\tilde{t}_{a}} S^{b}=\frac{\left[S^{b}, S^{a}\right]}{\left(z_{a}-z_{b}\right)^{2}}+\frac{\left[\eta^{a}, S^{b}\right]}{z_{a}-z_{b}} .
\end{aligned}
$$

These equation generalize the Heisenberg model which appears from (4.8) in $n=1$ (1-site) case:

$$
\partial_{t} S+\frac{k^{2}}{4 \lambda^{2}}\left[S, S_{x x}\right]=0
$$

and described by the Hamiltonian

$$
\mathcal{H}=\frac{k^{2}}{16 \lambda^{2}} \oint_{\mathbb{S}^{1}} \mathrm{~d} x\left\langle\left(\partial_{x} S\right)^{2}\right\rangle
$$

\subsection{1+1 XYZ Gaudin magnet: interacting Landau-Lifshitz models}

By analogy with the previous section the Hamiltonians in $0+1 \operatorname{sl}(2, \mathbb{C})$ case:

$$
\tilde{H}_{a}=\frac{1}{4}\left\langle S^{a} \hat{\wp}\left(S^{a}\right)\right\rangle+\frac{1}{2} \sum_{c \neq a}\left\langle S^{a} \hat{F}\left(S^{c}\right)\right\rangle-\frac{1}{4}\left\langle\left(\sum_{c \neq a} \hat{\varphi}_{a c}\left(S^{c}\right)\right)^{2}\right\rangle+\frac{H_{a}^{2}}{2 \lambda_{a}^{2}} .
$$

Consider now the following L-A pair:

$$
\begin{aligned}
& L(z)=\sum_{c=1}^{n} \sum_{\alpha=1}^{3} S_{\alpha}^{c} \sigma_{\alpha} \varphi_{\alpha}\left(z-z_{c}\right) \\
& \tilde{M}_{a}(z)=\sum_{\alpha=1}^{3} \eta_{\alpha}^{a} \sigma_{\alpha} \varphi_{\alpha}\left(z-z_{a}\right)+S_{\alpha}^{a} \sigma_{\alpha} \varphi_{\beta}\left(z-z_{a}\right) \varphi_{\gamma}\left(z-z_{a}\right),
\end{aligned}
$$

where $\alpha, \beta, \gamma$ are different indices equivalent to $1,2,3$ up to a cyclic permutation and (compare with $(4.6))^{6}$

$$
\eta^{a}=-\frac{k}{4 \lambda_{a}^{2}}\left[S^{a}, S_{x}^{a}\right]+\sum_{c \neq a} \hat{\varphi}_{a c}\left(S^{c}\right)+\frac{H_{a}}{\lambda_{a}^{2}} S^{a} .
$$

The zero curvature equation (4.7) leads to equations of motion:

$$
\begin{aligned}
& \partial_{\tilde{t}_{a}} S^{a}-k \partial_{x} \eta^{a}=\left[S^{a}, \hat{\wp}\left(S^{a}\right)\right]+\sum_{c \neq a}\left[\eta^{a}, \hat{\varphi}_{c a}\left(S^{c}\right)\right]-\hat{\varphi}_{c a}\left(\left[S^{c}, \hat{\varphi}_{c a}\left(S^{a}\right)\right]\right), \\
& \partial_{\tilde{t}_{a}} S^{b}=\left[\hat{\varphi}_{a b}\left(\eta^{a}\right), S^{b}\right]+\hat{\varphi}_{b a}\left(\left[\hat{\varphi}_{b a}\left(S^{b}\right), S^{a}\right]\right)
\end{aligned}
$$

\footnotetext{
${ }^{6}$ The remark about the term $\frac{H_{a}}{\lambda_{a}^{2}} S^{a}$ in the previous section is reasonable here as well.
} 
and $\eta^{a}(4.11)$ is a particular solution of the equation

$$
-k S_{x}^{a}=\left[S^{a}, \eta^{a}-\sum_{c \neq a} \hat{\varphi}_{a c}\left(S^{c}\right)\right] .
$$

It is fixed if we require $\eta^{a} \rightarrow \eta^{a}=\sum_{c \neq a} \hat{\varphi}_{a c}\left(S^{c}\right)+\frac{H_{a}}{\lambda_{a}^{2}} S^{a}$ in $(0+1)$ limit. The proof follows from (C.1)-(C.7). In particular, from (C.5) it follows that $\hat{\varphi}_{c a}\left(\left[S^{c}, \hat{\varphi}_{c a}\left(S^{a}\right)\right]\right)=\left[\hat{F}_{c a}\left(S^{c}\right), S^{a}\right]$. Then (4.2) is written in the form close to (3.6):

$$
\begin{aligned}
& \partial_{\tilde{t}_{a}} S^{a}-k \partial_{x} \eta^{a}=\left[S^{a}, \hat{\wp}\left(S^{a}\right)\right]+\sum_{c \neq a}\left[\eta^{a}, \hat{\varphi}_{c a}\left(S^{c}\right)\right]-\left[\hat{F}_{c a}\left(S^{c}\right), S^{a}\right], \\
& \partial_{\tilde{t}_{a}} S^{b}=\left[\hat{\varphi}_{a b}\left(\eta^{a}\right), S^{b}\right]+\left[S^{b}, \hat{F}_{b a}\left(S^{a}\right)\right] .
\end{aligned}
$$

The last three terms in the first equation of (3.6) vanish. It is due to (C.3) that $\forall A, B \in$ $\operatorname{sl}(2, \mathbb{C})$ :

$$
\hat{E}_{1}([A, B])=\left[\hat{E}_{1}(A), B\right]+\left[A, \hat{E}_{1}(B)\right] .
$$

Note that in case $n=1$ the first one equation in (4.13) is the Landau-Lifshitz equation:

$$
\partial_{t} S+\frac{k^{2}}{4 \lambda^{2}}\left[S, S_{x x}\right]=[S, \hat{\wp}(S)]
$$

described by the Hamiltonian

$$
\mathcal{H}=\oint_{\mathbb{S}^{1}} \mathrm{~d} x\left(\frac{1}{4}\langle S \hat{\wp}(S)\rangle+\frac{k^{2}}{16 \lambda^{2}}\left\langle\left(\partial_{x} S\right)^{2}\right\rangle\right) .
$$

\subsection{Hamiltonian description}

The explicit form of the conserved quantities in terms of the fields are obtained by solving the Riccati equation. First, we make the gauge transformation (see for example [19, 32]):

$$
\left(k \partial_{x}+\left(\begin{array}{cc}
L_{11} & L_{12} \\
L_{21} & -L_{11}
\end{array}\right)\right)\left(\begin{array}{l}
\psi_{1} \\
\psi_{2}
\end{array}\right)=0 \rightarrow\left(k \partial_{x}+\left(\begin{array}{cc}
0 & 1 \\
T & 0
\end{array}\right)\right)\left(\begin{array}{l}
\psi_{1} \\
\psi_{2}
\end{array}\right)=0
$$

with

$$
T=L_{12} L_{21}+L_{11}^{2}+k L_{11} \frac{\partial_{x} L_{12}}{L_{12}}-k \partial_{x} L_{11}-\frac{k^{2}}{2} \frac{\partial_{x}^{2} L_{12}}{L_{12}}+\frac{3 k^{2}}{4}\left(\frac{\partial_{x} L_{12}}{L_{12}}\right)^{2},
$$

which leads to the Schrödinger equation:

$$
\left(-k^{2} \partial_{x}^{2}+T\right) \psi_{1}=0
$$

Taking wave function in the form $\psi_{1}=e^{\frac{1}{k} \int_{x_{0}}^{x} \mathrm{~d} y \chi(y)}$ we come to the Riccati equation:

$$
k \partial_{x} \chi+\chi^{2}-T=0 .
$$

The solution is obtained via local decompositions:

$$
\chi_{a}=\frac{1}{z-z_{a}} \chi_{a,-1}+\chi_{a, 0}+\left(z-z_{a}\right) \chi_{a, 1}+\cdots,
$$




$$
\begin{aligned}
T & =\frac{1}{\left(z-z_{a}\right)^{2}} T_{a,-2}+\frac{1}{z-z_{a}} T_{a,-1}+T_{a, 0}+\cdots, \\
L & =\frac{1}{z-z_{a}} L^{a,-1}+L^{a, 0}+\left(z-z_{a}\right) L^{a, 1}+\cdots .
\end{aligned}
$$

Then (4.15) gives:

$$
\begin{aligned}
& \chi_{a,-1}^{2} \equiv \lambda_{a}^{2}=T_{a,-2}, \\
& \chi_{a, 0}=\frac{1}{2 \chi_{a,-1}}\left(T_{a,-1}-k \partial_{x} \chi_{a,-1}\right), \\
& \chi_{a, 1}=\frac{1}{2 \chi_{a,-1}}\left(T_{a, 0}-\frac{T_{a,-1}^{2}}{4 T_{a,-2}}\right),
\end{aligned}
$$

As it was shown in [32] $\chi_{a, k}(4.19)$ are the densities of the conservation laws. We will use notation

$$
h_{a, k}(x)=-\lambda_{a} \chi_{a, k-1}
$$

for the densities and

$$
\mathcal{H}_{a, k}=\oint_{\mathbb{S}^{1}} \mathrm{~d} x h_{a, k}(x)
$$

for the Hamiltonians. The coefficients (4.18) of the decomposition of $L$-matrices in rational and elliptic cases:

$$
\begin{aligned}
L^{a,-1} & =S^{a}, & L^{a,-1} & =S^{a}, \\
L^{a, 0} & =\sum_{c \neq a} \frac{S^{c}}{z_{a}-z_{c}}, & L^{a, 0} & =\sum_{c \neq a} \hat{\varphi}_{a c}\left(S^{c}\right), \\
L^{a, 1} & =-\sum_{c \neq a} \frac{S^{c}}{\left(z_{a}-z_{c}\right)^{2}}, & L^{a, 1} & =-\frac{1}{2} \hat{\wp}\left(S^{a}\right)-\sum_{c \neq a} \hat{F}_{a c}\left(S^{c}\right),
\end{aligned}
$$

In what follows we sometimes omit for the simplicity the index $a$ for $L$-matrix and its elements assuming decompositions (4.16)-(4.18). Substituting (4.18) into (4.14) we get:

$$
\begin{aligned}
T_{a,-2}= & L_{12}^{-1} L_{21}^{-1}+L_{11}^{-1} L_{11}^{-1} \\
T_{a,-1}= & L_{12}^{-1} L_{21}^{0}+L_{12}^{0} L_{21}^{-1}+2 L_{11}^{0} L_{11}^{-1}+k \frac{L_{11}^{-1} \partial_{x} L_{12}^{-1}}{L_{12}^{-1}}-k \partial_{x} L_{11}^{-1}, \\
T_{a, 0}= & L_{12}^{1} L_{21}^{-1}+L_{12}^{-1} L_{21}^{1}+2 L_{11}^{1} L_{11}^{-1}+L_{12}^{0} L_{21}^{0}+L_{11}^{0} L_{11}^{0} \\
& +\frac{k}{L_{12}^{-1}}\left(L_{11}^{0} \partial_{x} L_{12}^{-1}+L_{11}^{-1} \partial_{x} L_{12}^{0}-\frac{L_{12}^{0} L_{11}^{-1} \partial_{x} L_{12}^{-1}}{L_{12}^{-1}}\right)-k \partial_{x} L_{11}^{0} \\
& -\frac{k^{2}}{2} \frac{\partial_{x}^{2} L_{12}^{-1}}{L_{12}^{-1}}+\frac{3 k^{2}}{4}\left(\frac{\partial_{x} L_{12}^{-1}}{L_{12}^{-1}}\right) .
\end{aligned}
$$

Let us summarize the obtained results. 
Lemma 4.1. The density of the Hamiltonian $\chi_{a, 0}$ has the following form in terms of the decomposition of the L-matrix (4.18):

$$
2 \lambda_{a} \chi_{a, 0}=T_{a,-1}=\left\langle L^{-1} L^{0}\right\rangle-2 P_{a}=-2\left(H_{a}+P_{a}\right),
$$

where $P_{a}$ is the density of the Hamiltonian of the shift operator along $x$ corresponding to the $a^{\text {th }}$ site (marked point):

$$
\left\{\oint_{\mathbb{S}^{1}} d x P_{a}(x), S^{b}(y)\right\}=k \delta_{a b} \partial_{y} S^{a}, \quad P_{a}(x)=-\frac{k}{2} L_{11}^{a,-1}(x) \frac{\partial_{x} L_{12}^{a,-1}(x)}{L_{12}^{a,-1}(x)} .
$$

Proof. The nontrivial part of the proof is related to the shift operators $P_{a}$. Brackets (4.5) have the following form in standard basis (here we omit index $a$ as above):

$$
\begin{aligned}
& \left\{L_{12}^{-1}(x), L_{11}^{-1}(y)\right\}=2 L_{12}^{-1}(x) \delta(x-y), \quad\left\{L_{21}^{-1}(x), L_{11}^{-1}(y)\right\}=-2 L_{21}^{-1}(x) \delta(x-y), \\
& \left\{L_{12}^{-1}(x), L_{21}^{-1}(y)\right\}=-4 L_{11}^{-1}(x) \delta(x-y) .
\end{aligned}
$$

For example, let us verify that $\oint_{\mathbb{S}^{1}} \mathrm{~d} x\left\{L_{11}^{-1}(x) \frac{\partial_{x} L_{12}^{-1}(x)}{L_{12}^{-1}(x)}, L_{21}^{-1}(y)\right\}=-2 \partial_{y} L_{21}^{-1}(y)$ :

$$
\begin{aligned}
\oint_{\mathbb{S}^{1}} \mathrm{~d} x & \left\{L_{11}^{-1}(x) \frac{\partial_{x} L_{12}^{-1}(x)}{L_{12}^{-1}(x)}, L_{21}^{-1}(y)\right\} \\
= & \oint_{\mathbb{S}^{1}} \mathrm{~d} x\left\{L_{11}^{-1}(x), L_{21}^{-1}(y)\right\} \frac{\partial_{x} L_{12}^{-1}(x)}{L_{12}^{-1}(x)}-\oint_{\mathbb{S}^{1}} \mathrm{~d} x \partial_{x} L_{11}^{-1}(x)\left\{\ln L_{12}^{-1}(x), L_{21}^{-1}(y)\right\} \\
= & 2 L_{21}^{-1}(y) \frac{\partial_{y} L_{12}^{-1}(y)}{L_{12}^{-1}(y)}+4 L_{11}^{-1}(y) \frac{\partial_{y} L_{11}^{-1}(y)}{L_{12}^{-1}(y)}=-2 \partial_{y} L_{21}^{-1}(y) .
\end{aligned}
$$

The later follows from the condition $\partial_{y} \lambda_{a}^{2}=0=\partial_{y}\left(\left(L_{11}^{-1}(y)\right)^{2}+L_{12}^{-1}(y) L_{21}^{-1}(y)\right)$. The verification of (4.21) for other components can be performed in the same way.

Lemma 4.2. The density of the Hamiltonian $\chi_{a, 1}$ has the following form in terms of the decomposition of the L-matrix (4.18):

$$
\begin{aligned}
8 \lambda_{a}^{3} \chi_{a, 1}= & 4 \lambda_{a}^{2} T_{a, 0}-T_{a,-1}^{2} \\
= & 2\left\langle L^{a,-1} L^{a,-1}\right\rangle\left(\frac{1}{2}\left\langle L^{a, 0} L^{a, 0}\right\rangle+\left\langle L^{a, 1} L^{a,-1}\right\rangle\right)-\left(\left\langle L^{a,-1} L^{a, 0}\right\rangle\right)^{2} \\
& +2 k\left\langle L^{a, 0} \partial_{x} L^{a,-1} L^{a,-1}\right\rangle-\frac{k^{2}}{2}\left\langle\left(\partial_{x} L^{a,-1}\right)^{2}\right\rangle .
\end{aligned}
$$

Proof. Our purpose is to show that $\oint_{\mathbb{S}^{1}} \mathrm{~d} x$ l.h.s. $(4.22)=\oint_{\mathbb{S}^{1}} \mathrm{~d} x$ r.h.s. (4.22). The proof is direct. It is based on the integration by parts. For example,

$$
\begin{array}{rl}
\oint_{\mathbb{S} 1} \mathrm{~d} x & 4 \lambda_{a}^{2}\left(-\frac{k^{2}}{2} \frac{\partial_{x}^{2} L_{12}^{-1}}{L_{12}^{-1}}+\frac{3 k^{2}}{4}\left(\frac{\partial_{x} L_{12}^{-1}}{L_{12}^{-1}}\right)\right)-k^{2}\left(\frac{L_{11}^{-1} \partial_{x} L_{12}^{-1}}{L_{12}^{-1}}-\partial_{x} L_{11}^{-1}\right)^{2} \\
= & -\oint_{\mathbb{S}^{1}} \mathrm{~d} x \frac{k^{2}}{2}\left\langle L_{x}^{-1} L_{x}^{-1}\right\rangle
\end{array}
$$

and

$$
\begin{aligned}
& \oint_{\mathbb{S}^{1}} \mathrm{~d} x 4 \lambda_{a}^{2} \frac{k}{L_{12}^{-1}}\left(L_{11}^{0} \partial_{x} L_{12}^{-1}+L_{11}^{-1} \partial_{x} L_{12}^{0}-\frac{L_{12}^{0} L_{11}^{-1} \partial_{x} L_{12}^{-1}}{L_{12}^{-1}}\right) \\
& -2 k\left(\frac{L_{11}^{-1} \partial_{x} L_{12}^{-1}}{L_{12}^{-1}}-\partial_{x} L_{11}^{-1}\right)\left(L_{12}^{-1} L_{21}^{0}+L_{12}^{0} L_{21}^{-1}+2 L_{11}^{0} L_{11}^{-1}\right)=2 k \oint_{\mathbb{S}^{1}} \mathrm{~d} x\left\langle L^{0} L_{x}^{-1} L^{-1}\right\rangle .
\end{aligned}
$$


Finally, we have the following densities of the Hamiltonians describing the first and the second flows:

$$
\begin{aligned}
& h_{a, 1}=-\lambda_{a} \chi_{a, 0}=-\frac{1}{2} T_{a,-1}=P_{a}-\frac{1}{2}\left\langle L^{a,-1} L^{a, 0}\right\rangle, \\
& h_{a, 2}=-\lambda_{a} \chi_{a, 1}=-\frac{1}{2}\left(T_{a, 0}-\frac{1}{4 \lambda_{a}^{2}} T_{a,-1}^{2}\right)=-\frac{1}{4}\left\langle L^{a, 0} L^{a, 0}\right\rangle \\
& -\frac{1}{2}\left\langle L^{a, 1} L^{a,-1}\right\rangle+\frac{1}{8 \lambda_{a}^{2}}\left\langle L^{a,-1} L^{a, 0}\right\rangle^{2}-\frac{k}{4 \lambda_{a}^{2}}\left\langle L^{a, 0} \partial_{x} L^{a,-1} L^{a,-1}\right\rangle+\frac{k^{2}}{16 \lambda_{a}^{2}}\left\langle\left(\partial_{x} L^{a,-1}\right)^{2}\right\rangle .
\end{aligned}
$$

These equalities are understood in a sense that $\oint_{\mathbb{S}^{1}} \mathrm{~d} x$ l.h.s. $(4.23)=\oint_{\mathbb{S}^{1}} \mathrm{~d} x$ r.h.s. (4.23).

Theorem 4.1. The Hamiltonian densities (4.23) and the Poisson structure (4.5) provides equations (4.8) and (4.13).

Proof. Substituting (4.20) into (4.23) we obtain explicit expressions for the Hamiltonians. In rational case:

$$
\begin{aligned}
\mathcal{H}_{a, 1}= & \oint_{\mathbb{S}^{1}} \mathrm{~d} x h_{a, 1}(x)=\oint_{\mathbb{S}^{1}} \mathrm{~d} x\left(P_{a}-\frac{1}{2} \sum_{c \neq a} \frac{\left\langle S^{a} S^{c}\right\rangle}{z_{a}-z_{c}}\right)=\oint_{\mathbb{S}^{1}} \mathrm{~d} x\left(P_{a}+H_{a}\right), \\
\mathcal{H}_{a, 2}= & \oint_{\mathbb{S}^{1}} \mathrm{~d} x h_{a, 2}(x) \\
= & \oint_{\mathbb{S}^{1}} \mathrm{~d} x\left(\frac{1}{2} \sum_{c \neq a} \frac{\left\langle S^{a} S^{c}\right\rangle}{\left(z_{a}-z_{c}\right)^{2}}-\frac{1}{4}\left\langle\left(\sum_{c \neq a} \frac{S^{c}}{z_{a}-z_{c}}\right)^{2}\right\rangle+\frac{1}{8 \lambda_{a}^{2}}\left(\sum_{c \neq a} \frac{\left\langle S^{a} S^{c}\right\rangle}{z_{a}-z_{c}}\right)^{2}\right. \\
& \left.-\frac{k}{4 \lambda_{a}^{2}} \sum_{c \neq a} \frac{\left\langle S^{c} \partial_{x} S^{a} S^{a}\right\rangle}{z_{a}-z_{c}}+\frac{k^{2}}{16 \lambda_{a}^{2}}\left\langle\left(\partial_{x} S^{a}\right)^{2}\right\rangle\right) \\
= & \oint_{\mathbb{S}^{1}} \mathrm{~d} x\left(\tilde{H}_{a}-\frac{k}{4 \lambda_{a}^{2}} \sum_{c \neq a} \frac{\left\langle S^{c} \partial_{x} S^{a} S^{a}\right\rangle}{z_{a}-z_{c}}+\frac{k^{2}}{16 \lambda_{a}^{2}}\left\langle\left(\partial_{x} S^{a}\right)^{2}\right\rangle\right)
\end{aligned}
$$

and in the elliptic case:

$$
\begin{aligned}
\mathcal{H}_{a, 1}= & \oint_{\mathbb{S}^{1}} \mathrm{~d} x h_{a, 1}(x)=\oint_{\mathbb{S}^{1}} \mathrm{~d} x\left(P_{a}-\frac{1}{2} \sum_{c \neq a}\left\langle S^{a} \hat{\varphi}_{a c}\left(S^{c}\right)\right\rangle\right)=\oint_{\mathbb{S}^{1}} \mathrm{~d} x\left(P_{a}+H_{a}\right), \\
\mathcal{H}_{a, 2}= & \oint_{\mathbb{S}^{1}} \mathrm{~d} x h_{a, 2}(x)=\oint_{\mathbb{S}^{1}} \mathrm{~d} x\left(\frac{1}{4}\left\langle S^{a} \hat{\wp}\left(S^{a}\right)\right\rangle+\frac{1}{2} \sum_{c \neq a}\left\langle S^{a} \hat{F}\left(S^{c}\right)\right\rangle-\frac{1}{4}\left\langle\left(\sum_{c \neq a} \hat{\varphi}_{a c}\left(S^{c}\right)\right)^{2}\right\rangle\right. \\
& \left.+\frac{1}{8 \lambda_{a}^{2}}\left(\sum_{c \neq a}\left\langle S^{a} \hat{\varphi}_{a c}\left(S^{c}\right)\right\rangle\right)^{2}-\frac{k}{4 \lambda_{a}^{2}} \sum_{c \neq a}\left\langle\hat{\varphi}_{a c}\left(S^{c}\right) \partial_{x} S^{a} S^{a}\right\rangle+\frac{k^{2}}{16 \lambda_{a}^{2}}\left\langle\left(\partial_{x} S^{a}\right)^{2}\right\rangle\right) \\
= & \oint_{\mathbb{S}^{1}} \mathrm{~d} x\left(\tilde{H}_{a}-\frac{k}{4 \lambda_{a}^{2}} \sum_{c \neq a}\left\langle\hat{\varphi}_{a c}\left(S^{c}\right) \partial_{x} S^{a} S^{a}\right\rangle+\frac{k^{2}}{16 \lambda_{a}^{2}}\left\langle\left(\partial_{x} S^{a}\right)^{2}\right\rangle\right),
\end{aligned}
$$

where in $H_{a}, \tilde{H}_{a}$ from (4.1), (4.2) and (4.10) $S^{a}=S^{a}(x)$ is assumed. The rest of the proof is simple. One should write equations of motion generated by the obtained Hamiltonians and the Poisson structure (4.5) and verify that they coincide with equations (4.8) and (4.13). This evaluation is direct. 


\section{A Elliptic functions}

\section{A.1 Basic definitions and properties}

Most of definitions are borrowed from [38, 39]. We assume that $q=\exp (2 \pi i \tau)$, where $\tau$ is the modular parameter of the elliptic curve $\Sigma_{\tau}$ which is realized as $\mathbb{C} / \Gamma_{\tau}, \Gamma_{\tau}=\mathbb{Z} \oplus \mathbb{Z} \tau$.

The basic element is the theta function $(\mathbf{e}=\exp 2 \pi \imath)$ :

$$
\begin{aligned}
\vartheta(z \mid \tau) & =q^{\frac{1}{8}} \sum_{n \in \mathbf{Z}}(-1)^{n} \mathbf{e}\left(\frac{1}{2} n(n+1) \tau+n z\right) \\
& =q^{\frac{1}{8}} e^{-\frac{i \pi}{4}}\left(e^{i \pi z}-e^{-i \pi z}\right) \prod_{n=1}^{\infty}\left(1-q^{n}\right)\left(1-q^{n} e^{2 i \pi z}\right)\left(1-q^{n} e^{-2 i \pi z}\right) .
\end{aligned}
$$

The Eisenstein functions

$$
E_{1}(z \mid \tau)=\partial_{z} \log \vartheta(z \mid \tau), \quad E_{1}(z \mid \tau) \sim \frac{1}{z}-2 \eta_{1} z
$$

where

$$
\eta_{1}(\tau)=\frac{3}{\pi^{2}} \sum_{m=-\infty}^{\infty} \sum_{n=-\infty}^{\infty^{\prime}} \frac{1}{(m \tau+n)^{2}}=\frac{24}{2 \pi i} \frac{\eta^{\prime}(\tau)}{\eta(\tau)}
$$

and $\eta(\tau)=q^{\frac{1}{24}} \prod_{n>0}\left(1-q^{n}\right)$ is the Dedekind function

$$
E_{2}(z \mid \tau)=-\partial_{z} E_{1}(z \mid \tau)=\partial_{z}^{2} \log \vartheta(z \mid \tau), \quad E_{2}(z \mid \tau) \sim \frac{1}{z^{2}}+2 \eta_{1} .
$$

The higher Eisenstein functions

$$
E_{j}(z)=\frac{(-1)^{j}}{(j-1) !} \partial^{(j-2)} E_{2}(z), \quad j>2
$$

It is easy to see that the even-numbered functions are even and the odd-numbered ones are odd:

$$
E_{2 k}(-z)=E_{2 k}(z), \quad E_{2 k+1}(-z)=-E_{2 k+1}(z) .
$$

Relation to the Weierstrass functions

$$
\begin{aligned}
& \zeta(z, \tau)=E_{1}(z, \tau)+2 \eta_{1}(\tau) z, \\
& \wp(z, \tau)=E_{2}(z, \tau)-2 \eta_{1}(\tau) .
\end{aligned}
$$

The next important function is

$$
\begin{aligned}
& \phi(u, z)=\frac{\vartheta(u+z) \vartheta^{\prime}(0)}{\vartheta(u) \vartheta(z)}, \\
& \phi(u, z)=\phi(z, u), \quad \phi(-u,-z)=-\phi(u, z) .
\end{aligned}
$$

It has a pole at $z=0$ and

$$
\phi(u, z)=\frac{1}{z}+E_{1}(u)+\frac{z}{2}\left(E_{1}^{2}(u)-\wp(u)\right)+\cdots .
$$


Let $f(u, z)=\partial_{u} \phi(u, z)$. Then

$$
f(u, z)=\phi(u, z)\left(E_{1}(u+z)-E_{1}(u)\right)
$$

Heat equation

$$
\partial_{\tau} \phi(u, w)-\frac{1}{2 \pi i} \partial_{u} \partial_{w} \phi(u, w)=0 .
$$

Quasi-periodicity

$$
\begin{array}{ll}
\vartheta(z+1)=-\vartheta(z), & \vartheta(z+\tau)=-q^{-\frac{1}{2}} e^{-2 \pi i z} \vartheta(z), \\
E_{1}(z+1)=E_{1}(z), & E_{1}(z+\tau)=E_{1}(z)-2 \pi i, \\
E_{2}(z+1)=E_{2}(z), & E_{2}(z+\tau)=E_{2}(z), \\
\phi(u, z+1)=\phi(u, z), & \phi(u, z+\tau)=e^{-2 \pi \imath u} \phi(u, z), \\
f(u, z+1)=f(u, z), & f(u, z+\tau)=e^{-2 \pi \imath u} f(u, z)-2 \pi \imath \phi(u, z) .
\end{array}
$$

The Fay three-section formula:

$$
\phi\left(u_{1}, z_{1}\right) \phi\left(u_{2}, z_{2}\right)-\phi\left(u_{1}+u_{2}, z_{1}\right) \phi\left(u_{2}, z_{2}-z_{1}\right)-\phi\left(u_{1}+u_{2}, z_{2}\right) \phi\left(u_{1}, z_{1}-z_{2}\right)=0 .
$$

Particular case of this formula is the Calogero functional equation

$$
\begin{aligned}
& \phi(u, z) \partial_{v} \phi(v, z)-\phi(v, z) \partial_{u} \phi(u, z)=\left(E_{2}(u)-E_{2}(v)\right) \phi(u+v, z), \\
& \phi(u, z) \phi(-u, z)=E_{2}(z)-E_{2}(u), \\
& \phi\left(z, u_{1}\right) \phi\left(z, u_{2}\right)=\phi\left(z, u_{1}+u_{2}\right)\left(E_{1}(z)+E_{1}\left(u_{1}\right)+E_{1}\left(u_{2}\right)-E_{1}\left(z+u_{1}+u_{2}\right)\right) .
\end{aligned}
$$

Another important relation is

$$
\begin{aligned}
& \phi(v, z-w) \phi\left(u_{1}-v, z\right) \phi\left(u_{2}+v, w\right)-\phi\left(u_{1}-u_{2}-v, z-w\right) \phi\left(u_{2}+v, z\right) \phi\left(u_{1}-v, w\right) \\
& \quad=\phi\left(u_{1}, z\right) \phi\left(u_{2}, w\right) f\left(u_{1}, u_{2}, v\right),
\end{aligned}
$$

where

$$
f\left(u_{1}, u_{2}, v\right)=E_{1}(v)-E_{1}\left(u_{1}-u_{2}-v\right)+E_{1}\left(u_{1}-v\right)-E_{1}\left(u_{2}+v\right) .
$$

Taking limit $u_{2} \rightarrow 0$ in (A.13) we obtain:

$$
\begin{aligned}
& \phi(v, z-w) \phi\left(u_{1}-v, z\right) \phi(v, w)-\phi\left(u_{1}-v, z-w\right) \phi(v, z) \phi\left(u_{1}-v, w\right) \\
& =\phi\left(u_{1}, z\right)\left(E_{2}(v)-E_{2}\left(u_{1}-v\right)\right)
\end{aligned}
$$

which is equivalent to (A.10) due to (A.7).

Theta functions with characteristics. For $a, b \in \mathbb{Q}$ by definition:

$$
\theta\left[\begin{array}{l}
a \\
b
\end{array}\right](z, \tau)=\sum_{j \in \mathbb{Z}} \mathbf{e}\left((j+a)^{2} \frac{\tau}{2}+(j+a)(z+b)\right) .
$$

In particular, the function $\vartheta(\mathrm{A} .1)$ is a theta function with characteristics:

$$
\vartheta(x, \tau)=\theta\left[\begin{array}{l}
1 / 2 \\
1 / 2
\end{array}\right](x, \tau) .
$$


Properties:

$$
\begin{aligned}
& \theta\left[\begin{array}{l}
a \\
b
\end{array}\right](z+1, \tau)=\mathbf{e}(a) \theta\left[\begin{array}{l}
a \\
b
\end{array}\right](z, \tau), \\
& \theta\left[\begin{array}{l}
a \\
b
\end{array}\right]\left(z+a^{\prime} \tau, \tau\right)=\mathbf{e}\left(-a^{\prime 2} \frac{\tau}{2}-a^{\prime}(z+b)\right) \theta\left[\begin{array}{c}
a+a^{\prime} \\
b
\end{array}\right](z, \tau), \\
& \theta\left[\begin{array}{c}
a+j \\
b
\end{array}\right](z, \tau)=\theta\left[\begin{array}{l}
a \\
b
\end{array}\right](z, \tau), \quad j \in \mathbb{Z} .
\end{aligned}
$$

The following notations are used: $\theta\left[\begin{array}{l}a / 2 \\ b / 2\end{array}\right](z)=\theta_{a b}(z)$. Then $\theta_{11}(z)=\vartheta(z)$ from (A.1).

\section{B Lie algebra $\operatorname{sl}(N, \mathbb{C})$ and elliptic functions}

Introduce the notation

$$
\mathbf{e}_{N}(z)=\exp \left(\frac{2 \pi i}{N} z\right)
$$

and two matrices

$$
\begin{aligned}
Q & =\operatorname{diag}\left(\mathbf{e}_{N}(1), \ldots, \mathbf{e}_{N}(m), \ldots, 1\right), \\
\Lambda & =\left(\begin{array}{ccccc}
0 & 1 & 0 & \cdots & 0 \\
0 & 0 & 1 & \cdots & 0 \\
\vdots & \vdots & \ddots & \ddots & \vdots \\
0 & 0 & 0 & \cdots & 1 \\
1 & 0 & 0 & \cdots & 0
\end{array}\right) .
\end{aligned}
$$

Let

$$
\Gamma_{N}=\mathbb{Z}_{N}^{(2)}=(\mathbb{Z} / N \mathbb{Z} \oplus \mathbb{Z} / N \mathbb{Z}), \quad \Gamma_{N}^{\prime}=\tilde{\mathbb{Z}}_{N}^{(2)}=\mathbb{Z}_{N}^{(2)} \backslash(0,0)
$$

be the two-dimensional lattices of orders $N^{2}$ and $N^{2}-1$ correspondingly. The generators of the lattice $\Gamma_{N}$ corresponding to the elliptic curve $\Sigma_{\tau}$ (generated by the lattice $\Gamma_{\tau}$ ) are $\frac{1}{N}$ and $\frac{1}{\tau}$. The matrices $Q^{a_{1}} \Lambda^{a_{2}}, a=\left(a_{1}, a_{2}\right) \in \mathbb{Z}_{N}^{(2)}$ generate a basis in the group $\operatorname{GL}(N, \mathbb{C})$, while $Q^{\alpha_{1}} \Lambda^{\alpha_{2}}$, $\alpha=\left(\alpha_{1}, \alpha_{2}\right) \in \tilde{\mathbb{Z}}_{N}^{(2)}$ generate a basis in the Lie algebra $\operatorname{sl}(N, \mathbb{C})$. Consider the projective representation of $\mathbb{Z}_{N}^{(2)}$ in $\mathrm{GL}(N, \mathbb{C})$

$$
\begin{aligned}
& a \rightarrow T_{a}=\mathbf{e}_{N}\left(\frac{a_{1} a_{2}}{2}\right) Q^{a_{1}} \Lambda^{a_{2}}, \\
& T_{a} T_{b}=\mathbf{e}_{N}\left(-\frac{a \times b}{2}\right) T_{a+b}, \quad\left(a \times b=a_{1} b_{2}-a_{2} b_{1}\right) .
\end{aligned}
$$

The natural Killing form is

$$
\left\langle T_{\alpha} T_{\beta}\right\rangle \stackrel{\text { def }}{=} \operatorname{Tr}\left(T_{\alpha} T_{\beta}\right)=N \delta_{0, \alpha+\beta} \bmod \Gamma_{N} .
$$

It follows from (B.5) that

$$
\left[T_{\alpha}, T_{\beta}\right]=c_{\alpha, \beta} T_{\alpha+\beta},
$$


where

$$
c_{\alpha, \beta}=2 \sqrt{-1} \sin \frac{\pi}{N}(\alpha \times \beta)
$$

are the structure constants of $\operatorname{sl}(N, \mathbb{C})$. They obey the following properties:

$$
c_{\alpha, \beta}=c_{\alpha, \beta+\alpha}, \quad c_{\alpha, \beta}=-c_{\beta, \alpha}=c_{\beta,-\alpha} .
$$

Introduce the following constants on $\tilde{\mathbb{Z}}^{(2)}$ :

$$
\begin{aligned}
& \vartheta\left(\omega_{\gamma}\right)=\vartheta\left(\frac{\gamma_{1}+\gamma_{2} \tau}{N}\right), \\
& E_{1}\left(\omega_{\gamma}\right)=E_{1}\left(\frac{\gamma_{1}+\gamma_{2} \tau}{N}\right), \quad E_{2}\left(\omega_{\gamma}\right)=E_{2}\left(\frac{\gamma_{1}+\gamma_{2} \tau}{N}\right), \quad \wp\left(\omega_{\gamma}\right)=\wp\left(\frac{\gamma_{1}+\gamma_{2} \tau}{N}\right)(B .
\end{aligned}
$$

and the quasi-periodic functions on $\Sigma_{\tau}$

$$
\begin{aligned}
& \phi_{\gamma}(z)=\phi\left(\frac{\gamma_{1}+\gamma_{2} \tau}{N}, z\right) \\
& \varphi_{\gamma}(z)=\mathbf{e}_{N}\left(\gamma_{2} z\right) \phi_{\gamma}(z) \\
& f_{\gamma}(z)=\left.\mathbf{e}_{N}\left(\gamma_{2} z\right) \partial_{u} \phi(u, z)\right|_{u=\frac{\gamma_{1}+\gamma_{2} \tau}{N}}=\varphi_{\gamma}(z)\left(E_{1}\left(\omega_{\gamma}+z\right)-E_{1}\left(\omega_{\gamma}\right)\right), \\
& F_{\gamma}(z)=\varphi_{\gamma}(z) E_{1}(z)-f_{\gamma}(z)=\varphi_{\gamma}(z)\left(E_{1}(z)+E_{1}\left(\omega_{\gamma}\right)-E_{1}\left(\omega_{\gamma}+z\right)\right) .
\end{aligned}
$$

Function $\varphi_{\gamma}(z)$ is an element of the basis in the space of sections with a simple pole at $z=0$ of the bundle $\operatorname{End}(V)$ for the holomorphic vector bundle $V$ of degree 1. It follows from (A.5) that

$$
\begin{array}{ll}
\varphi_{\gamma}(z+1)=\mathbf{e}_{N}\left(\gamma_{2}\right) \varphi_{\gamma}(z), & \varphi_{\gamma}(z+\tau)=\mathbf{e}_{N}\left(-\gamma_{1}\right) \varphi_{\gamma}(z), \\
f_{\gamma}(z+1)=\mathbf{e}_{N}\left(\gamma_{2}\right) f_{\gamma}(z), & f_{\gamma}(z+\tau)=\mathbf{e}_{N}\left(-\gamma_{1}\right) f_{\gamma}(z)-2 \pi \imath \varphi_{\gamma}(z), \\
F_{\gamma}(z+1)=\mathbf{e}_{N}\left(\gamma_{2}\right) F_{\gamma}(z), & F_{\gamma}(z+\tau)=\mathbf{e}_{N}\left(-\gamma_{1}\right) F_{\gamma}(z) .
\end{array}
$$

Function $F_{\gamma}(z)$ is the quasi-periodic and has the second order pole at $z=0$.

Let us write down the Fay-type formulae. It follows from (A.9)-(A.12) that

$$
\begin{aligned}
& \varphi_{\gamma}\left(z-z_{a}\right) \varphi_{\beta}\left(z-z_{c}\right) \stackrel{(\mathrm{A} .9)}{=} \varphi_{\beta+\gamma}\left(z-z_{a}\right) \varphi_{\beta}\left(z_{a}-z_{c}\right)+\varphi_{\beta+\gamma}\left(z-z_{c}\right) \varphi_{\gamma}\left(z_{c}-z_{a}\right), \\
& \varphi_{\beta}(z) f_{\gamma}(z)-\varphi_{\gamma}(z) f_{\beta}(z) \stackrel{\text { (A.10) }}{=} \varphi_{\beta+\gamma}(z)\left(\wp\left(\omega_{\beta}\right)-\wp\left(\omega_{\gamma}\right)\right), \\
& \varphi_{\alpha}(z) \varphi_{-\alpha}(z) \stackrel{\text { A.11) }}{=} \wp(z)-\wp\left(\omega_{\alpha}\right), \\
& \varphi_{\beta}(z) \varphi_{\gamma}(z) \stackrel{(\mathrm{A} .12)}{=} \varphi_{\beta+\gamma}(z)\left(E_{1}(z)+E_{1}\left(\omega_{\beta}\right)+E_{1}\left(\omega_{\gamma}\right)-E_{1}\left(z+\omega_{\beta}+\omega_{\gamma}\right)\right), \\
& \varphi_{\gamma}\left(z_{1}\right) \varphi_{\gamma}\left(z_{2}\right) \stackrel{\text { A.12) }}{=} \varphi_{\gamma}\left(z_{1}+z_{2}\right)\left(E_{1}\left(z_{1}\right)+E_{1}\left(z_{2}\right)+E_{1}\left(\omega_{\gamma}\right)-E_{1}\left(z_{1}+z_{2}+\omega_{\gamma}\right)\right) .
\end{aligned}
$$

The last one identity can be also rewritten (using (A.6) which is $\varphi_{\gamma}(-z)=-\varphi_{-\gamma}(z)$ and (A.2)) as follows:

$$
\varphi_{\gamma}\left(z_{1}\right) \varphi_{-\gamma}\left(z_{2}\right)=-\varphi_{\gamma}\left(z_{1}-z_{2}\right)\left(E_{1}\left(z_{1}\right)-E_{1}\left(z_{2}\right)+E_{1}\left(\omega_{\gamma}\right)-E_{1}\left(z_{1}-z_{2}+\omega_{\gamma}\right)\right) .
$$

We also need the following relation:

$$
\begin{aligned}
& -\varphi_{\beta}\left(z-z_{c}\right) f_{\gamma}\left(z-z_{a}\right)+\varphi_{\gamma}\left(z-z_{a}\right) f_{\beta}\left(z-z_{c}\right) \\
& \quad=-\varphi_{\beta+\gamma}\left(z-z_{c}\right) f_{\gamma}\left(z_{c}-z_{a}\right)+\varphi_{\beta+\gamma}\left(z-z_{a}\right) f_{\beta}\left(z_{a}-z_{c}\right) .
\end{aligned}
$$




\section{Lie algebra $\operatorname{sl}(2, \mathbb{C})$ and elliptic functions}

For $\operatorname{SL}(2, \mathbb{C})$ instead of $T_{\alpha}$ we use the basis of sigma-matrices

$$
\begin{aligned}
& \sigma_{0}=\mathrm{Id}, \quad \sigma_{1}=T_{0,1}, \quad \sigma_{2}=T_{1,1}, \quad \sigma_{3}=-T_{1,0}, \\
& \left\{\sigma_{a}\right\}=\left\{\sigma_{0}, \sigma_{\alpha}\right\}, \quad a=0, \alpha, \quad \alpha=1,2,3, \\
& \sigma_{+}=\frac{\sigma_{1}-\imath \sigma_{2}}{2}, \quad \sigma_{-}=\frac{\sigma_{1}+\imath \sigma_{2}}{2} .
\end{aligned}
$$

The standard theta-functions with the characteristics are

$$
\theta_{0,0}=\theta_{3}, \quad \theta_{1,0}=\theta_{2}, \quad \theta_{0,1}=\theta_{4}, \quad \theta_{1,1}=\theta_{1} .
$$

For $\alpha=1,2,3$ and $\left\{\omega_{\alpha}\right\}=\left\{\frac{\tau}{2}, \frac{\tau+1}{2}, \frac{1}{2}\right\}$

$$
\varphi_{\alpha}(z)=\mathbf{e}\left(z \partial_{\tau} \omega_{\alpha}\right) \frac{\vartheta^{\prime}(0) \vartheta\left(z+\omega_{\alpha}\right)}{\vartheta(z) \vartheta\left(\omega_{\alpha}\right)} .
$$

Table 1.

\begin{tabular}{|c||c|c|c|}
\hline$\alpha$ & $(1,0)$ & $(0,1)$ & $(1,1)$ \\
\hline$\sigma_{\alpha}$ & $\sigma_{3}$ & $\sigma_{1}$ & $\sigma_{2}$ \\
\hline half-periods & $\omega_{1}=\frac{1}{2}$ & $\omega_{2}=\frac{\tau}{2}$ & $\omega_{3}=\frac{1+\tau}{2}$ \\
\hline$\varphi_{\alpha}(z)$ & $\frac{\theta_{2}(z) \theta_{1}^{\prime}(0)}{\theta_{2}(0) \theta_{1}(z)}$ & $\frac{\theta_{4}(z) \theta_{1}^{\prime}(0)}{\theta_{4}(0) \theta_{1}(z)}$ & $\frac{\theta_{3}(z) \theta_{1}^{\prime}(0)}{\theta_{3}(0) \theta_{1}(z)}$ \\
\hline
\end{tabular}

In $\operatorname{sl}(2, \mathbb{C})$ case some more properties appear in addition to the previously listed. In what follows $\alpha, \beta, \gamma$ are different indices equivalent to 1, 2, 3 up to a cyclic permutation. Then $\omega_{\alpha}+\omega_{\beta}=\omega_{\gamma} \bmod \Gamma_{2}$ and $\varphi_{\alpha+\beta}(z)=\varphi_{\gamma}(z)$,

$$
\begin{aligned}
& \varphi_{-\alpha}(z)=\varphi_{\alpha}(z), \quad \varphi_{\alpha}(-z)=-\varphi_{\alpha}(z), \\
& E_{1}\left(\omega_{\alpha}\right)=-2 \pi \sqrt{-1} \partial_{\tau} \omega_{\alpha} .
\end{aligned}
$$

Indeed, from (A.2) and (A.8) we have $-E_{1}\left(\frac{\tau}{2}\right)=E_{1}\left(\frac{\tau}{2}-\tau\right)=E_{1}\left(\frac{\tau}{2}\right)+2 \pi \sqrt{-1}$. Then

$$
\begin{aligned}
& E_{1}\left(\omega_{\alpha}\right)+E_{1}\left(\omega_{\beta}\right)=E_{1}\left(\omega_{\alpha}+\omega_{\beta}\right), \\
& \left(\varphi_{\alpha}(z)\right)^{2}=\wp(z)-\wp\left(\omega_{\alpha}\right) .
\end{aligned}
$$

For small $z$ :

$$
\varphi_{\alpha}(z)=\frac{1}{z}-\frac{z}{2} \wp\left(\omega_{\alpha}\right)+\cdots .
$$

From (C.3) we also have

$$
F_{\alpha}(z)=\varphi_{\beta}(z) \varphi_{\gamma}(z)=-\partial_{z} \varphi_{\alpha}(z) .
$$

The Fay identity (A.9) reads:

$$
\varphi_{\gamma}\left(z-z_{a}\right) \varphi_{\beta}\left(z-z_{c}\right)=\varphi_{\alpha}\left(z-z_{a}\right) \varphi_{\beta}\left(z_{a}-z_{c}\right)-\varphi_{\alpha}\left(z-z_{c}\right) \varphi_{\gamma}\left(z_{a}-z_{c}\right) .
$$

Combining (C.6) we may get:

$$
\begin{aligned}
& \varphi_{\beta}\left(z-z_{c}\right) \varphi_{\beta}\left(z-z_{a}\right) \varphi_{\alpha}\left(z-z_{a}\right)=\varphi_{\beta}\left(z-z_{a}\right) \varphi_{\gamma}\left(z-z_{a}\right) \varphi_{\beta}\left(z_{a}-z_{c}\right) \\
& \quad+\varphi_{\alpha}\left(z-z_{c}\right) \varphi_{\alpha}\left(z_{c}-z_{a}\right) \varphi_{\beta}\left(z_{c}-z_{a}\right)-\varphi_{\alpha}\left(z-z_{a}\right) \varphi_{\alpha}\left(z_{c}-z_{a}\right) \varphi_{\gamma}\left(z_{c}-z_{a}\right)
\end{aligned}
$$

or

$$
\begin{aligned}
& \varphi_{\gamma}\left(z-z_{c}\right) \varphi_{\alpha}\left(z-z_{a}\right) \varphi_{\gamma}\left(z-z_{a}\right)=\varphi_{\beta}\left(z-z_{a}\right) \varphi_{\gamma}\left(z-z_{a}\right) \varphi_{\gamma}\left(z_{a}-z_{c}\right) \\
& \quad+\varphi_{\alpha}\left(z-z_{a}\right) \varphi_{\alpha}\left(z_{c}-z_{a}\right) \varphi_{\beta}\left(z_{a}-z_{c}\right)-\varphi_{\alpha}\left(z-z_{c}\right) \varphi_{\alpha}\left(z_{c}-z_{a}\right) \varphi_{\gamma}\left(z_{a}-z_{c}\right) .
\end{aligned}
$$




\section{Acknowledgements}

Author is grateful to M.A. Olshanetsky for useful discussions and remarks. The work was supported by grants RFBR-09-02-00393, RFBR-09-01-92437-KEa, RFBR-09-01-93106-NCNILa, Russian President fund MK-1646.2011.1 and to the Federal Agency for Science and Innovations of Russian Federation under contract 14.740.11.0347.

\section{References}

[1] Gaudin M., Diagonalisation dùne classe d'Hamiltoniens de spin, J. Physique 37 (1976), 1087-1098. Gaudin M., La fonction d'onde de Bethe, Masson, Paris, 1983 (in French); Mir, Moscow, 1987 (in Russian).

[2] Baxter R.J., One-dimensional anisotropic Heisenberg chain, Ann. Physics 70 (1972), 323-337.

[3] Sklyanin E.K., Takebe T., Algebraic bethe ansatz for the XYZ Gaudin model, Phys. Lett. A 219 (1996), 217-225, q-alg/9601028.

[4] Zotov A., Elliptic linear problem for Calogero-Inozemtsev model and Painlevé VI equation, Lett. Math. Phys. 67 (2004), 153-165, hep-th/0310260.

Levin A., Olshanetsky M., Zotov A., Painlevé VI, rigid tops and reflection equation, Comm. Math. Phys. 268 (2006), 67-103, math.QA/0508058.

[5] Levin A., Zotov A., On rational and elliptic forms of Painlevé VI equation, Amer. Math. Soc. Transl. Ser. 2, Vol. 221, Amer. Math. Soc., Providence, RI, 173-184.

[6] Fuchs R., Über lineare homogene Differentialgleichungen zweiterordnung mit im endlich gelegne wesentlich singulären Stellen, Math. Ann. 63 (1907), 301-323.

Schlesinger L., Über eine Klasse von Differentialsystemen beliebiger Ordnung mit festen kritischen Punkten, J. Reine Angew. Math. 141 (1912), 96-145.

[7] Reyman A.G., Semenov-Tian-Shansky M.A., Lie algebras and Lax equations with spectral parameter on an elliptic curve, Zap. Nauchn. Semin. Leningr. Otd. Mat. Inst. Steklova 150 (1986), 104-118 (in Russian).

[8] Belavin A.A., Drinfel'd V.G., Solutions of the classical Yang-Baxter equation for simple Lie algebras, Funktsional. Anal. i Prilozhen. 16 (1982), no. 3, 1-29 (English transl.: Funct. Anal. Appl. 16 (1982), 159-180).

[9] Zotov A.V., Levin A.M.; Olshanetsky M.A., Chernyakov Yu.B., Quadratic algebras related to elliptic curves, Theoret. and Math. Phys. 156 (2008), 1103-1122, arXiv:0710.1072.

[10] Sklyanin E.K., Separation of variables in the Gaudin model, Zap. Nauchn. Sem. Leningrad. Otdel. Mat. Inst. Steklov. (LOMI) 164 (1987), 151-169 (English transl.: J. Soviet Math. 47 (1989), 2473-2488).

Sklyanin E.K., Takebe T., Separation of variables in the elliptic Gaudin model, Comm. Math. Phys. 204 (1999), 17-38, solv-int/9807008.

[11] Sklyanin E.K., Generating function of correlators in the $\mathrm{sl}_{2}$ Gaudin model, Lett. Math. Phys. 47 (1999), 275-292, solv-int/9708007.

Takasaki K., Gaudin model, KZ equation and an isomonodromic problem on the tours, Lett. Math. Phys. 44 (1998), 143-156, hep-th/9711058.

Feigin B., Frenkel E., Reshetikhin N., Gaudin model, Bethe ansatz and critical level, Comm. Math. Phys. 166 (1994), 27-62, hep-th/9402022.

Gould M.D., Zhang Y.-Z., Zhao S.-Y., Elliptic Gaudin models and elliptic KZ equations, Nuclear Phys. B 630 (2002), 492-508, nlin.SI/0110038.

Chernyakov Yu., Levin A., Olshanetsky M., Zotov A., Elliptic Schlesinger system and Painlevé VI, J. Phys. A: Math. Gen. 39 (2006), 12083-12101, nlin.SI/0602043.

[12] Khesin B., Levin A., Olshanetsky M., Bihamiltonian structures and quadratic algebras in hydrodynamics and on non-commutative torus, Comm. Math. Phys. 250 (2004), 581-612, nlin.SI/0309017.

[13] Petrera M., Suris Yu.B., An integrable discretization of the rational $\mathfrak{s u}(2)$ Gaudin model and related systems, Comm. Math. Phys. 283 (2008), 227-253, arXiv:0707.4088.

Ragnisco O., Zullo F., Bäcklund transformations for the trigonometric Gaudin magnet, SIGMA 6 (2010), 012, 6 pages, arXiv:0912.2456.

Veselov A.P., What is an integrable mapping?, in What is Integrability?, Editor V.E. Zakharov, Springer Ser. Nonlinear Dynam., Springer, Berlin, 1991, 251-272.

Hone A.N.W., Kuznetsov V.B., Ragnisco O., Bäcklund transformations for the sl(2) Gaudin magnet, J. Phys. A: Math. Gen. 34 (2001), 2477-2490, nlin.SI/0007041. 
[14] Talalaev D.V., The quantum Gaudin system, Funktsional. Anal. i Prilozhen. 40 (2006), no. 1, 86-91 (English transl.: Funct. Anal. Appl. 40 (2006), 73-77).

Rubtsov V., Silantyev A., Talalaev D., Manin matrices, quantum elliptic commutative families and characteristic polynomial of elliptic Gaudin model, SIGMA 5 (2009), 110, 22 pages, arXiv:0908.4064.

[15] Frenkel E., Affine algebras, Langlands duality and Bethe ansatz, q-alg/9506003.

Chervov A., Talalaev D., Quantum spectral curves, quantum integrable systems and the geometric Langlands correspondence, hep-th/0604128.

Teschner J., Quantization of the Hitchin moduli spaces, Liouville theory, and the geometric Langlands correspondence I, arXiv:1005.2846.

[16] Enriquez B., Rubtsov V., Hitchin systems, higher Gaudin operators and R-matrices, Math. Res. Lett. 3 (1996), 343-357, alg-geom/9503010.

[17] Hitchin N., Stable bundles and integrable systems, Duke Math. J. 54 (1987), 91-114.

[18] Nekrasov N., Holomorphic bundles and many-body systems, Comm. Math. Phys. 180 (1996), 587-603, hepth/9503157.

Gorsky A., Nekrasov N., Elliptic Calogero-Moser system from two dimensional current algebra, hepth/9401021.

[19] Levin A., Olshanetsky M., Zotov A., Hitchin systems - symplectic Hecke correspondence and twodimensional version, Comm. Math. Phys. 236 (2003), 93-133, nlin.SI/0110045.

[20] Petrera M., Ragnisco O., From su(2) gaudin models to integrable tops, SIGMA 3 (2007), 058, 14 pages, math-ph/0703044.

Levin A., Zotov A., An integrable system of interacting elliptic tops, Teoret. Mat. Fiz. 146 (2006), 55-64 (English transl.: Theoret. and Math. Phys. 146 (2006), 45-52).

[21] Kuznetsov V.B., Isomorphism of the $n$-dimensional Neumann system and the $n$-site Gaudin magnet, Funktsional. Anal. i Prilozhen. 26 (1992), no. 4, 88-90 (English transl.: Funct. Anal. Appl. 26 (1992), 302-304).

[22] Zakharov V.E., Shabat A.B., A scheme for integrating the nonlinear equations of mathematical physics by the method of the inverse scattering problem. I, Funktsional. Anal. i Prilozhen. 8 (1974), no. 3, 43-53 (English transl.: Funct. Anal. Appl. 8 (1974), 226-235).

[23] Faddeev L., Takhtajan L., Hamiltonian approach to solitons theory, Nauka, Moscow, 1986 (in Russian).

[24] Dubrovin B.A., Krichever I.M., Novikov S.P., Integrable systems. I, Current Problems in Mathematics. Fundamental Directions, Vol. 4, Itogi Nauki i Tekhniki, Akad. Nauk SSSR, Vsesoyuz. Inst. Nauchn. i Tekhn. Inform., Moscow, 1985, 179-284 (in Russian).

[25] Sokolov V.V., Schabat A.B., Classification of integrable evolution equations, Soviet Sci. Rev. Sect. C Math. Phys. Rev., Vol. 4, Harwood Academic Publ., Chur, 1984, 221-280.

Mikhailov A.V., Schabat A.B., Yamilov R.I., The symmetry approach to classification of nonlinear equations. Complete list of integrable systems, Uspekhi Mat. Nauk 42 (1987), no. 4, 3-53 (English transl.: Russian Math. Surveys 42 (1987), no. 4, 1-63).

Fokas A.S., Symmetries and integrability, Stud. Appl. Math. 77 (1987), 253-299.

[26] Landau L., Lifshitz E., On the theory of the dispersion of magnetic permeability in ferromagnetic bodies, Phys. Zeitsch. der Sow. 8 (1935), 153-169.

[27] Sklyanin E., On complete integrability of the Landau-Lifshitz equation, Preprint LOMI E-3-79, 1979. Borovik A.E., Robuk V.N., Linear pseudopotentials and conservation laws for the Landau-Lifshits equation describing the nonlinear dynamics of a ferromagnet with uniaxial anisotropy, Teoret. Mat. Fiz. 46 (1981), 371-381 (English transl.: Theoret. and Math. Phys. 46 (1981), 242-248).

[28] Baxter R.J., Eight-vertex model in lattice statistics and one-dimensional anisotropic Heisenberg chain. I. Some fundamental eigenvectors, Ann. Physics 76 (1973), 1-24.

Takhtajan L.A., Faddeev L.D., The quantum method of the inverse problem and the Heisenberg XYZ model, Uspekhi Mat. Nauk 34 (1979), no. 5, 13-63 (English transl.: Russian Math. Surveys 34 (1979), no. 5, 11-68).

[29] Zakharov V.E., Mikhailov A.V., Relativistically invariant two-dimensional models of field theory which are integrable by means of the inverse scattering problem method, Soviet Phys. JETP 74 (1978), 1953-1973.

Pohlmeyer K., Integrable Hamiltonian systems and interactions through quadratic constraints, Comm. Math. Phys. 46 (1976), 207-221.

[30] Cherednik I.V., Local conservation laws of principal chiral fields (d=1), Teoret. Mat. Fiz. 38 (1979), 179185 (English transl.: Theoret. and Math. Phys. 38 (1979), 120-124).

Cherednik I.V., Relativistically invariant quasiclassical limits of integrable two-dimensional quantum models, Teoret. Mat. Fiz. 47 (1981), 225-229 (English transl.: Theoret. and Math. Phys. 47 (1981), 422-425). 
[31] Krichever I., Vector bundles and Lax equations on algebraic curves, Comm. Math. Phys. 229 (2002), 229269, hep-th/0108110.

[32] Dubrovin B.A., Matveev V.B., Novikov S.P., Non-linear equations of Korteweg-de Vries type, finite-zone linear operators, and Abelian varieties, Uspekhi Mat. Nauk 31 (1976), no. 1, 55-136 (English transl.: Russian Math. Surveys 31 (1976), no. 1, 59-146).

[33] Golubchik I.Z., Sokolov V.V., Multicomponent generalization of the hierarchy of the Landau-Lifshitz equation, Teoret. Mat. Fiz. 124 (2000), 62-71 (English transl.: Theoret. and Math. Phys. 124 (2000), 909-917).

[34] Akhmetshin A.A., Krichever I.M., Volvovski Yu.S., Elliptic families of solutions of the KadomtsevPetviashvili equation, and the field analogue of the elliptic Calogero-Moser system, Funktsional. Anal. $i$ Prilozhen. 36 (2002), no. 4, 1-17 (English transl.: Funct. Anal. Appl. 36 (2002), 253-266), hep-th/0203192.

[35] Skrypnik T., 'Doubled' generalized Landau-Lifshiz hierarchies and special quasigraded Lie algebras, J. Phys. A: Math. Gen. 37 (2004), 7755-7768.

Skrypnik T., Quasigraded Lie algebras and matrix generalization of Landau-Lifshitz equation, in Proceedinds of Fifth International Conference "Symmetry in Nonlinear Mathematical Physics" (June 23-29, 2003, Kyiv), Editors A.G. Nikitin, V.M. Boyko, R.O. Popovych and I.A. Yehorchenko, Proceedings of Institute of Mathematics, Kyiv 50 (2004), Part 1, 462-469.

[36] Holod P.I., The hidden symmetry of the Landau-Lifshits equation, the hierarchy of higher equations and a dual equation for an asymmetric chiral field, Teoret. Mat. Fiz. 70 (1987), 18-29 (English transl.: Theoret. and Math. Phys. 70 (1987), 11-19).

[37] Orlov A.Yu., $N$-soliton solution of chiral fields on Grassmann manifolds ( $\sigma$ model), Teoret. Mat. Fiz. 61 (1984), 214-225 (English transl.: Theoret. and Math. Phys. 61 (1984), 1099-1107).

[38] Weyl A., Elliptic functions according to Eisenstein and Kronecker, Ergebnisse der Mathematik und ihrer Grenzgebiete, Band 88, Springer-Verlag, Berlin - New York, 1976.

[39] Mumford D., Tata lectures on theta. I, Progress in Mathematics, Vol. 28, Birkhäuser Boston, Inc., Boston, MA, 1983.

Mumford D., Tata lectures on theta. II. Jacobian theta functions and differential equations, Progress in Mathematics, Vol. 43, Birkhäuser Boston, Inc., Boston, MA, 1984. 